Review

\title{
A Numerical and Graphical Review of Energy Storage Technologies
}

\author{
Siraj Sabihuddin *, Aristides E. Kiprakis and Markus Mueller \\ Institute for Energy Systems (IES), School of Engineering, University of Edinburgh, \\ Faraday Building, King's Buildings, Mayfield Road, Edinburgh EH9 3JL, UK; \\ E-Mails: aristides.kiprakis@ed.ac.uk (A.E.K.); markus.mueller@ed.ac.uk (M.M.) \\ * Author to whom correspondence should be addressed; E-Mail: s.sabihuddin@ed.ac.uk; \\ Tel.: +44-0-131-650-6487; Fax: +44-0-131-650-6554.
}

Academic Editor: Izumi Taniguchi

Received: 18 November 2014 / Accepted: 12 December 2014 / Published: 29 December 2014

\begin{abstract}
More effective energy production requires a greater penetration of storage technologies. This paper takes a looks at and compares the landscape of energy storage devices. Solutions across four categories of storage, namely: mechanical, chemical, electromagnetic and thermal storage are compared on the basis of energy/power density, specific energy/power, efficiency, lifespan, cycle life, self-discharge rates, capital energy/power costs, scale, application, technical maturity as well as environmental impact. It's noted that virtually every storage technology is seeing improvements. This paper provides an overview of some of the problems with existing storage systems and identifies some key technologies that hold promise.
\end{abstract}

Keywords: energy; power; storage; density; specific; efficiency; lifespan; discharge; cost; scale

\section{Introduction}

The accelerated growth of the modern energy economy is highly dependent (Figure 1) on potentially dwindling fossil fuel resources. Some predictions indicate that depletion of known reserves are expected within 34-40 years for oil, 106-200 years for coal and 36-70 years for natural gas [1]. These predictions have spurred a renewed interest in renewable generation technologies. Technologies range from our 
traditional wind turbines, solar panels, hydroelectric, biomass and geothermal systems to emerging tidal and wave generators. Improvements in renewables have become a driving force for improved energy storage and have highlighted the necessity for storage even for non-renewables. Energy storage may be essential for a transition to sustainable energy production. In this paper, we provide a brief summary of storage methods and how they compare with each other. The review covers the fairly wide landscape of storage solutions as shown in Figure 2.
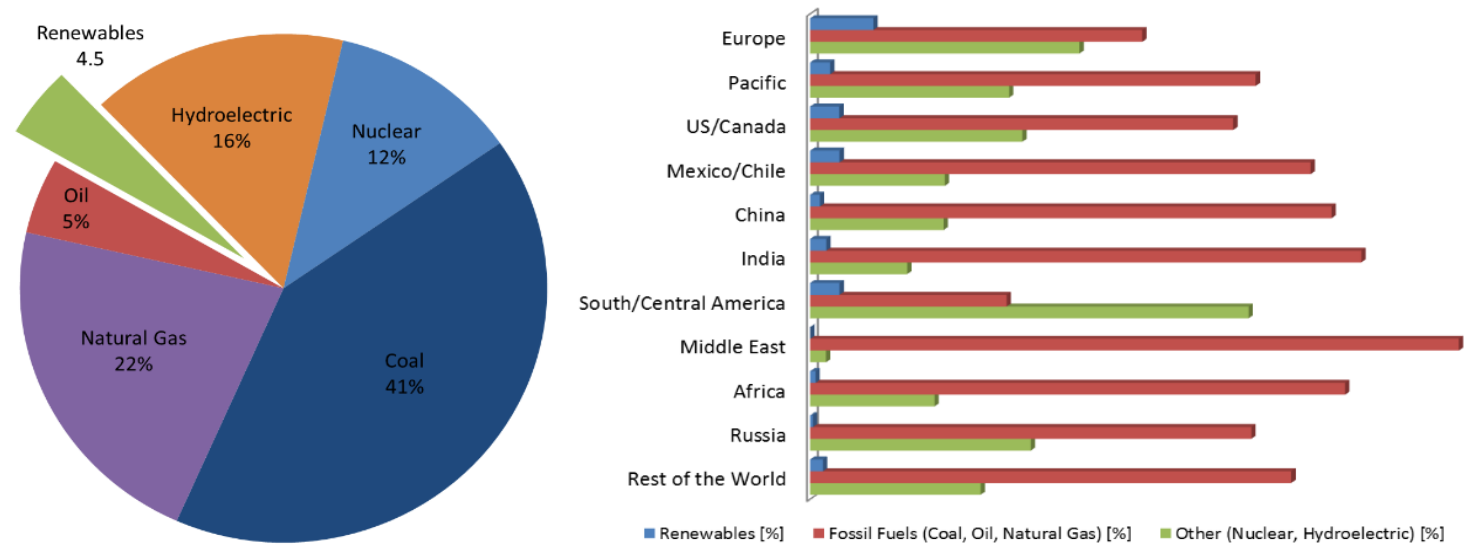

Figure 1. On the left, worldwide distribution of electricity production in 2011. On the right, national reliance for electricity production as of 2010. Data extracted from [2,3].

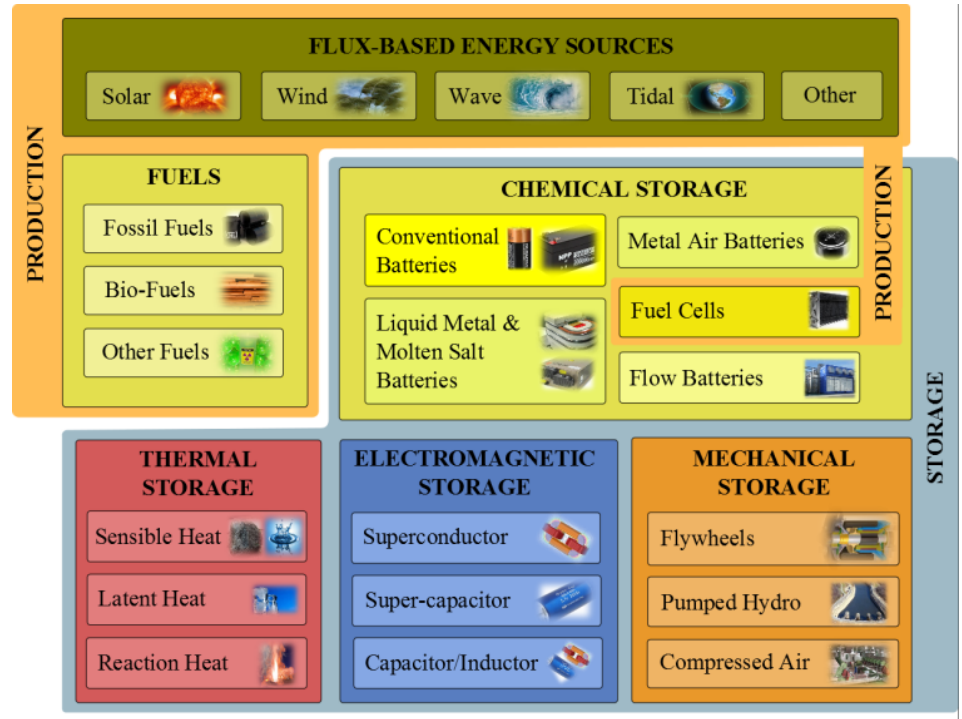

Figure 2. The landscape of energy/electricity generation and storage solutions (representative sample). It should be noted that fuel cells are a production technology but have been categorized as storage to adhere to common convention.

\subsection{Energy Storage Technologies Overview}

Energy storage allows de-coupling of energy production from consumption, thus decreasing the necessity for constant monitoring and prediction of consumer peak energy demands (Figure 3). Storage provides economic benefits by allowing a reduction of plant energy production to meet average demands rather than peak demands. Transmission lines and equipment can also be appropriately sized for average 
power demands. In addition, it also mitigates some problems associated with the intermittency of renewable energy generation.
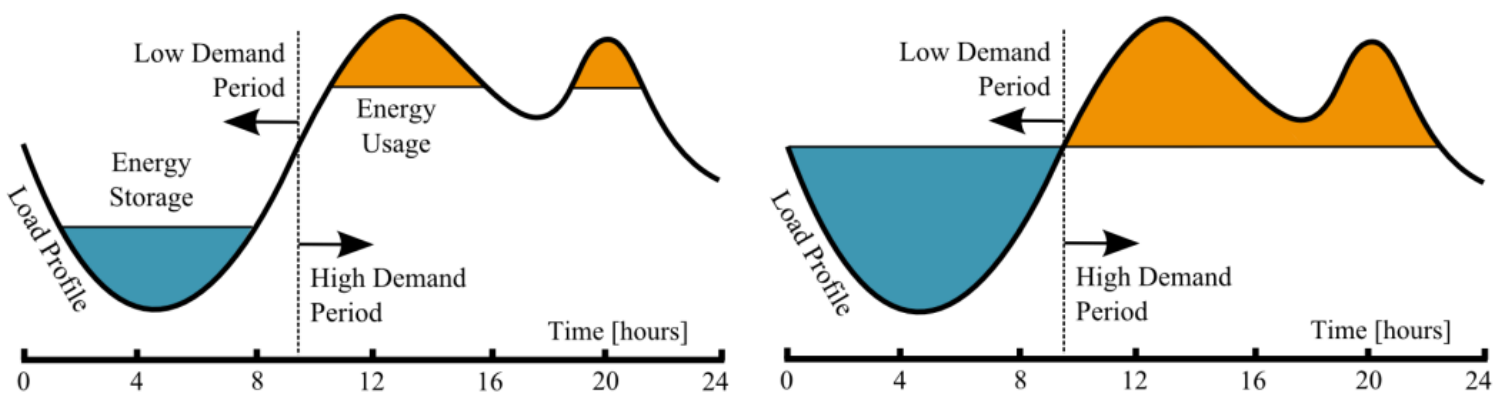

Figure 3. Consumer energy demands (load profile) over a $24 \mathrm{~h}$ period. The figure on the left shows a typical application of energy storage. While other intermediate applications exist, arguably, the ultimate goal of energy storage is load leveling as shown on the right. Figure modified from [4].

As we saw in Figure 2, a wide range of storage technologies exist. They can be broadly divided into four categories: mechanical, chemical, electromagnetic and thermal storage. Currently, however, the storage landscape is primarily dominated by mechanical storage in the form of pumped hydroelectric. In-fact, pumped hydroelectric storage accounts for around 99\% (127,000 MW) of all currently deployed forms of energy storage - this is followed by compressed air (440 MW), sodium sulphur (316 MW), lead acid (35 MW), nickel cadmium (27 MW), flywheel (25 MW), lithium ion (20 MW) and flow battery (3 MW) technologies [5,6].

This paper discusses not only pumped hydro but also compares the wider range of alternative storage options. Table 1 shows these potential applications in terms of power capacity while Table 2 shows categorizations based on response time and storage duration.

Technologies with high parasitic losses, such as, flywheels and superconductors, tend to be more useful for very short duration applications in power quality and regulation. As parasitic losses drop (e.g., pumped hydro), devices become more useful for longer term energy management. In the coming sections, we look at the positive and negative aspects of each class of device. We conclude by identifying key issues and likely future trends in the energy storage landscape.

Table 1. A categorization of scales of energy storage and their applications.

\begin{tabular}{|c|c|c|}
\hline Category & Applications & Power Rating \\
\hline Small Scale & Mobile Devices, Electric Vehicles, Satellites, etc. & $\leq 1 \mathrm{MW}$ \\
\hline Medium Scale & Office buildings, Remote communities, etc. & $10-100 \mathrm{MW}$ \\
\hline Large Scale & Power Plants, etc. & $\geq 300 \mathrm{MW}$ \\
\hline
\end{tabular}


Table 2. Typical applications of energy storage based on storage duration. Data obtained from multiple sources [7].

\begin{tabular}{|c|c|c|c|}
\hline Category & \multicolumn{2}{|l|}{ Applications } & Storage Duration \\
\hline \multirow{9}{*}{$\begin{array}{c}\text { Power Quality \& } \\
\text { Regulation }\end{array}$} & Fluctuation Suppression/Smoothing & $\mathrm{FS} / \mathrm{S}$ & \multirow{9}{*}{$\leq 1 \mathrm{~min}$} \\
\hline & Dynamic Power Response & DPR & \\
\hline & Low Voltage Ride Through & LVRT & \\
\hline & Line Fault Ride Through & LFRT & \\
\hline & Uninterruptable Power Supply & UPS & \\
\hline & Voltage Control Support & VCS & \\
\hline & Reactive Power Control & $\mathrm{RPC}$ & \\
\hline & Oscillation Damping & OD & \\
\hline & Transient Stability & TS & \\
\hline & Spinning/Contingency Reserves & $\mathrm{S} / \mathrm{CR}$ & \\
\hline & Ramping & $\mathrm{R}$ & \\
\hline Bridging Power & Emergency Backup & EB & $1 \mathrm{~min}-1 \mathrm{~h}$ \\
\hline & Load Following & LF & \\
\hline & Wind Power Smoothing & WPS & \\
\hline & Peak Shaving/Generation/Time Shifting & $\mathrm{PS} / \mathrm{G} / \mathrm{TS}$ & $1-10 \mathrm{~h}$ \\
\hline & Transmission Curtailment & TC & \\
\hline & Energy Arbitrage & EA & \\
\hline & Transmission \& Distribution Deferral & TDD & $5-12 \mathrm{~h}$ \\
\hline & Line Repair & LR & $5-1211$ \\
\hline & Load Cycling & $\mathrm{LC}$ & \\
\hline Energy Management & Weather Smoothing & WS & \\
\hline & Unit Commitment & $\mathrm{UC}$ & \\
\hline & Load Leveling & LL & hourc dout \\
\hline & Capacity Firming & $\mathrm{CF}$ & nours-aays \\
\hline & Renewable Integration and Backup & RIB & \\
\hline & Seasonal Storage & SS & $>4 \quad>>$ \\
\hline & Annual Smoothing & AS & 11001 \\
\hline
\end{tabular}

\subsection{Comments on Presented Data}

Before proceeding, it should be noted that technology comparisons are made with respect to energy, power, efficiency, lifespan, cycle life, self-discharge rate, scale and cost. Application, technical maturity and environmental impacts are also considered. Additionally, when necessary, discharge has been assumed to occur at a $1 \mathrm{C}$ rate. Data has been collected/computed from multiple sources a complete listing of which has been made available online at [7]. In order to build a measure of confidence, every data metric for every technology has been sampled from multiple sources - the range, standard deviation $(\sigma)$, mean $(\mu)$, median $(\tilde{x})$ and sample size $(n)$ values have been indicated to demonstrate the general distribution of quoted numbers across literature. Attempts to avoid theoretical and projected numbers have been made. Specific power and energy are computed by taking into account dry mass of the devices in question. In the case of fuel cells, operation over a $24 \mathrm{~h}$ time span has been used for comparison-it should be noted however, that these are, typically, used as production devices not storage devices. 
Volume based densities are computed by taking into account specific device dimensions. Scale data considers not only single devices but real world examples of energy farms - in some cases such wide scale deployment hasn't occurred and so scale estimates tend to be quite small. In the absence of data some assumptions are made about the maximum efficiency of devices. Cost comparisons have been obtained from recent sources within the last 5-10 years without present value adjustments and with the assumption that exchange rates have remained relatively stable.

\section{Mechanical Energy Storage}

Mechanical storage takes the form of either potential energy or kinetic energy storage. Pumped Hydroelectric, compressed air and flywheel systems are the best known and are compared in Table 3 and Figure 4.

Table 3. Comparing mechanical storage based on fourteen different metrics. Data obtained or calculated from multiple sources [7].

\begin{tabular}{|c|c|c|c|c|}
\hline \multirow{2}{*}{\multicolumn{2}{|c|}{ Metric }} & Pumped Hydro. & Compressed Air & Flywheel \\
\hline & & range $\sigma / \mu / \tilde{x} / n$ & range $\sigma / \mu / \tilde{x} / n$ & range $\sigma / \mu / \tilde{x} / n$ \\
\hline Specific Energy & {$[\mathrm{Wh} / \mathrm{Kg}]$} & $\begin{array}{c}0.30-1.33 \\
0.48 / 0.87 / 0.78 / 6\end{array}$ & $\begin{array}{c}3.20-60.00 \\
19.89 / 28.50 / 30.00 / 11 \\
\end{array}$ & $\begin{array}{c}5.00-200.00 \\
56.94 / 58.16 / 30.00 / 19\end{array}$ \\
\hline Energy Density & {$\left[\mathrm{KWh} / \mathrm{m}^{3}\right]$} & $\begin{array}{c}0.50-1.33 \\
0.45 / 1.07 / 1.15 / 6 \\
\end{array}$ & $\begin{array}{c}0.40-20.00 \\
6.65 / 7.19 / 5.00 / 9 \\
\end{array}$ & $\begin{array}{c}0.25-424.00 \\
137.36 / 95.16 / 20.00 / 12 \\
\end{array}$ \\
\hline Specific Power & {$[\mathrm{W} / \mathrm{Kg}]$} & $\begin{array}{c}0.01-0.12 \\
0.05 / 0.05 / 0.04 / 4 \\
\end{array}$ & $\begin{array}{c}2.20-24.0 \\
12.10 / 16.13 / 22.20 / 3\end{array}$ & $\begin{array}{c}400.00-30,000.00 \\
8,631 / 6,592 / 3,250 / 12 \\
\end{array}$ \\
\hline Power Density & {$\left[\mathrm{KW} / \mathrm{m}^{3}\right]$} & $\begin{array}{c}0.01-0.12 \\
0.05 / 0.05 / 0.04 / 4\end{array}$ & $\begin{array}{c}0.04-10.00 \\
3.81 / 3.03 / 1.90 / 7\end{array}$ & $\begin{array}{c}40.00-2,000.00 \\
814.40 / 816.29 / 707.00 / 7\end{array}$ \\
\hline Efficiency & {$[\%]$} & $\begin{array}{c}65.00-87.00 \\
7.13 / 76.59 / 80.00 / 17\end{array}$ & $\begin{array}{c}57.00-89.00 \\
12.28 / 68.30 / 70.00 / 23\end{array}$ & $\begin{array}{c}70.00-96.00 \\
6.31 / 89.36 / 90.00 / 22\end{array}$ \\
\hline Lifespan & {$[\mathrm{yr}]$} & $\begin{array}{c}20.00-80.00 \\
23.14 / 49.20 / 45.00 / 12\end{array}$ & $\begin{array}{c}20.00-40.00 \\
8.66 / 30.00 / 30.00 / 9\end{array}$ & $\begin{array}{c}15.00-20.00 \\
2.89 / 17.50 / 17.50 / 4\end{array}$ \\
\hline Cycle Life & [cycles] & $\begin{array}{c}10,000-60,000 \\
19,070 / 29,000 / 20,000 / 7\end{array}$ & $\begin{array}{c}8,000-30,000 \\
9,712 / 16,000 / 12,000 / 7\end{array}$ & $\begin{array}{c}10,000-100,000 \\
35,667 / 41,100 / 20,500 / 10\end{array}$ \\
\hline $\begin{array}{c}\text { Self-Discharge } \\
\text { Rate }\end{array}$ & {$[\% /$ day $]$} & $\begin{array}{c}0.00 \\
0.00 / 0.00 / 0.00 / 5\end{array}$ & $\begin{array}{c}0.00 \\
0.00 / 0.00 / 0.00 / 4\end{array}$ & $\begin{array}{c}24.00-100.00 \\
39.92 / 64.61 / 72.00 / 7\end{array}$ \\
\hline Scale & {$[\mathrm{MW}]$} & $\begin{array}{c}10.00-8,000.00 \\
1,998 / 1,542 / 800 / 28\end{array}$ & $\begin{array}{c}0.01-3,000.00 \\
792.14 / 467.72 / 150 / 33\end{array}$ & $\begin{array}{c}0.001-10.00 \\
4.62 / 1.96 / 0.20 / 25\end{array}$ \\
\hline $\begin{array}{c}\text { Energy Capital } \\
\text { Cost }\end{array}$ & [US\$/KWh] & $\begin{array}{c}1.00-291.20 \\
66.51 / 57.94 / 33.00 / 19\end{array}$ & $\begin{array}{c}1.00-140.00 \\
41.41 / 35.67 / 11.88 / 18\end{array}$ & $\begin{array}{c}200.00-150,000.00 \\
31,071 / 12,454 / 900 / 26\end{array}$ \\
\hline $\begin{array}{c}\text { Power Capital } \\
\text { Cost }\end{array}$ & {$[\mathrm{US} \$ / \mathrm{KW}]$} & $\begin{array}{c}300.00-5,288.00 \\
1,133 / 1,414 / 1,000 / 25\end{array}$ & $\begin{array}{c}400.00-2,250.00 \\
412.19 / 649.55 / 500 / 21\end{array}$ & $\begin{array}{c}30.28-700.00 \\
149.29 / 296.14 / 290.0 / 16\end{array}$ \\
\hline \multicolumn{2}{|c|}{ Application } & $\begin{array}{c}\text { Very Large Scale } \\
\text { Energy Management }\end{array}$ & $\begin{array}{c}\text { Very Large Scale } \\
\text { Energy Management }\end{array}$ & $\begin{array}{l}\text { Medium Scale } \\
\text { Power Quality }\end{array}$ \\
\hline \multicolumn{2}{|c|}{ Technical Maturity } & $\begin{array}{c}\text { Very Mature/ } \\
\text { Fully Commercialized }\end{array}$ & Proven/Commercializing & Mature/Commercializing \\
\hline \multicolumn{2}{|c|}{ Environmental Impact } & High/Medium & Medium/Low & Very Low \\
\hline
\end{tabular}




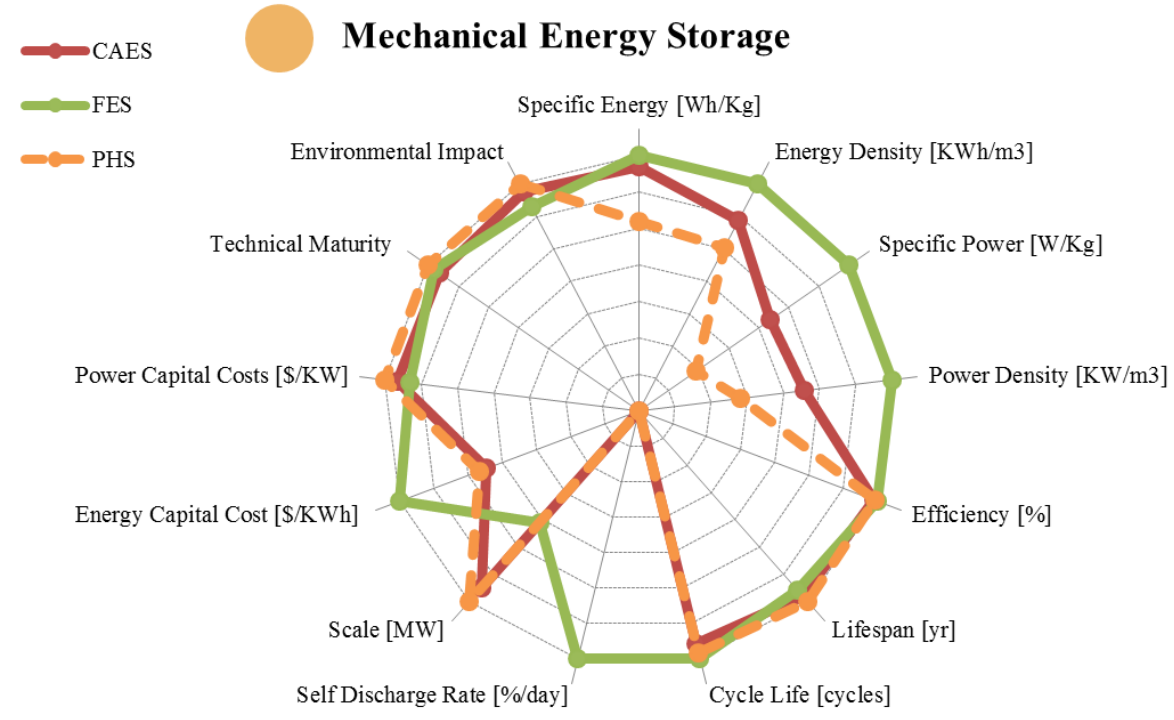

Figure 4. Comparing mechanical energy storage using normalized and logarithmically plotted average data from Table 3. PHS, shown as a dashed line is used as a basis of comparison for all other energy storage technologies.

\subsection{Pumped Hydroelectric Energy Storage (PHS)}

Pumped hydroelectric systems (PHS) are the dominant form of energy storage in the world today and are really the only feasible and commercially proven means of storing massive quantities of energy for relatively long durations [8,9]. Largest installed capacities are in the USA $(21.8 \mathrm{GW})$ and Japan (24.6 GW) followed by much smaller installations by European countries like Spain (5.3 MW). There are over 300 installed PHS systems worldwide [9]. These installations take two main forms: Conventional (over-ground) and underground. Some systems are considered pure PHS while others are pump-back systems. Pure PHS utilizes two reservoirs an upper and lower one. A relatively level head race conducts water to a vertical penstock and down through a turbine to the lower reservoir and into a raised tail race for pumping [10]. Pump-back PHS is more common and typically consists of a single reservoir. Pump-back systems are usually augmentations to traditional hydroelectric generation stations [9]. As a result they are more economically attractive and have uses related to base load generation (especially in times of excess flow). They are also used for irrigation and flood control as well. As pump-back systems operate in a similar regime to other base-load generation facilities, it has been suggested that comparisons to plants such as open cycle gas turbines would be useful [9].

PHS is highly reliable, flexible and can be used for up-down regulation of power as well as frequency stabilization [8,9]. Systems have long life, very low self-discharge rates, low operation and maintenance costs and high round trip efficiencies [9,10]. Traditionally, PHS has been used to complement high inertia base load generation applications (e.g., nuclear) [11]. Recent regulations and government targets have resulted in renewed interest in PHS. This is especially true in the context of intermittent renewables such as wind [9]. The quick/black start facility of PHS makes it particularly useful for these applications. Price arbitrage and reserve power are the primary income sources followed by black start and reactive power control. Energy security has often been cited as a reason for continuing investment in PHS [12]. 
Unfortunately, PHS is often associated with high capital cost, long development time, long pay-back periods and uncertain profitability [10,12]. Traditionally, PHS investment has been spurred by the need for peaking power for base-load generation stations or by periods of increased fossil fuel prices [11]. Where applicable such investments are done on a case-by-case basis due to high local parameter variability [11]. The highest costs are associated with land acquisition, civil works, steel structures and mechanical/electrical machinery. Further costs are incurred to help mitigate environmental impacts, comply with local regulations, pay for transmission line, equipment and water usage fees [12]. Financial concerns have been the primary reason for projects being dropped. Site availability is also a point of concern especially given that two adjacent reservoirs with good geotechnical conditions and high head height must be located or built $[9,10]$. Profitable topographies are difficult to find - the best of these are nearing exhaustion $[8,10]$. Even with appropriate topography, as we see from Table 3 , the low density of water means that energy density remains low. Non-adjacent reservoirs will result in further viscosity related losses too [10].

Local resistance to new PHS projects has often been encountered. These groups have concerns over impacts including diversion of river flows, creation of artificial water bodies, water quality, power and transmission line routing, bad smells, mosquito plagues, bursting dam risks and earthquake risks [11-13]. Furthermore, construction inconvenience and time can be a point of contention despite monetary compensation [12]. Some concerns, however, are a result of lack of awareness [11].

To combat other local and environmental concerns there has been a shift towards underground PHS. These systems use abandoned mine shafts (i.e., coal), quarries, ground water systems or other caverns composed of competent rock formations (with lower deformation and erosion) to store water and associated pumping and generating machinery. This approach also reduces the noise associated with PHS operation [12]. The relative fixed cost of underground reservoir excavation makes them more lucrative financially since storage capacity grows linearly with depth. Excavated soil can be used to form very strong burst resistant upper reservoir dykes and dams [10]. Availability of underground sites is likely to be higher and utilization of such sites has less impact on fish ecologies [11]. A full one quarter of future proposed PHS projects appear to be of the underground variety. Some have suggested that offshore sites may be an option for storage-unfortunately, only one such PHS project has been undertaken and corrosion prevention has been the main point of focus [9]. Further studies analyzing losses in PHS systems need to be systematically undertaken [10]. Despite the negatives, PHS facilities continue to be improved. Variable speed pumps and turbines have been introduced for greater frequency regulation control and for operation at optimal efficiencies [9]. Turbine blades have also seen some improvements [14]. One particularly interesting and particularly novel alternative has been proposed to increase energy densities in PHS: Hydraulic hydro energy storage. The approach utilizes water pressure to lift up a very large excavated rock cylinder the same way water is lifted - unfortunately this approach has a number of issues (tectonic movements, maintaining water tight seals, friction) and has yet to see any experimental work [15].

\subsection{Compressed Air Energy Storage (CAES)}

Compressed air energy storage (CAES) is the second most viable contender for large scale storage after PHS. It can be used for long or short duration storage at many scales. CAES systems utilize air that 
undergoes three main processing steps: Compression, storage and expansion [16]. Research has focused on improving one or more of these three steps. In some sense, CAES can be considered a mature technology and many variants resemble peaking gas turbine power plants. Despite the maturity of this technology, very few commercial implementations have been seen to present day and so economic viability remains in question.

CAES operates under a similar regime to that of PHS and has many of the same advantages and applications, i.e., high reliability, flexibility, long life, relatively low operation and maintenance costs and low self-discharge rates. In addition, as we see from Table 3, CAES also shows higher volumetric and mass energy and power outputs in comparison to PHS [17]. CAES profits are highly variable and economic feasibility is high primarily when natural gas prices are low and electricity prices high [18]. CAES alone is not an answer to energy storage but, like PHS, it can significantly offset investment on power plant capacity.

Smaller scale systems $(<100 \mathrm{KW})$ utilize pressurized tanks while larger ones use underground caverns for storage. Like PHS, the geological suitability of the storage site remains a significant issue and is the primary factor affecting cost [16]. Storage sites can take the form of solution mined domal salt caves, abandoned natural gas wells, hard rock/limestone mines, aquifiers or surface/buried air tanks. Domal salt caves can take two years to create but have fairly low environmental impacts [19]. Natural gas wells aren't created to withstand fast variations in pressure so tend to be a poor choice. Likewise air tanks are costly. As a result porous rock aquifiers with sealed cap rocks have become the most lucrative and cheapest CAES storage sites [16]. Some hybrid offshore CAES systems have been proposed. These systems are cost effective only if storage has high efficiency. Unfortunately the difficulty of finding appropriate storage sites (i.e., in undersea underground caverns) and the difficulties of operating gas turbines in such locations makes these hybrid systems unattractive [20]. Some hybrid-wind approaches store excess energy in pressure vessels (without the need for gas turbines) prior to electrical generation to down-size expensive electrical components [17]. Cryogenic storage systems may even cool air or another gas down to a low volume liquid phase - this fuel is then stored in thermally insulated chambers for release in gaseous phase through a turbine to generate electricity.

During storage, rapid air compression increases thermal energy which is lost to the ambient surroundings. The resulting loss in efficiency has been a key point of focus for recent work [20]. Maintaining isothermal temperature conditions is one way to minimize thermal losses and improve efficiency. Liquid pistons utilize conformable fluids and inject water droplets to improve heat transfer characteristics and maintain isothermal conditions [21]. Porous materials or rods may be inserted for even further improvements [16,17]. Multi-stage compressors are sometimes used with inter- and after-coolers to help keep air temperature low and reduce thermal stress on cavern walls during storage. Increases in the number of compression stages generate efficiencies approaching those of adiabatic conditions [16]. Isothermal pumping requires slow pumping to maintain constant temperature during compression. However, the compression cycle can be combined with thermal energy storage (discussed later) to save the generated heat from fast compression for later use during the expansion cycle [22]. Some advanced adiabatic approaches also utilize materials such as zeolite to adsorb air from the cavern or storage tank and reduce pressures and compression needed for storage [16]. Most such advanced techniques remain in design stages to present day. 
Compressed air is pre-heated, mixed with natural gas and ignited through a gas turbine to produce energy. Most existing research focuses on improvements in this expansion phase [16]. Unlike traditional gas turbine power plants, expansion occurs at a separate time from compression resulting in significant fuel savings. Some additional energy (via a recuperator) is put into pre-heating stored air to prevent chilling and brittling of turbine blades and to improve efficiency of combustion [20]. Storage of compression phase heat in molten salts can reduce pre-heating resulting in round-trip efficiencies of 90\%-95\% efficiencies [16]. Ultimately though, PHS has two distinct advantages over CAES. For one, while $\mathrm{CO}_{2}$ emissions are very low, CAES generally (not always) burns natural gas or some other fuel. Second, CAES requires significant ramping times of up to 10 min to reach full capacity output.

\subsection{Flywheel Energy Storage (FES)}

Flywheel energy storage (FES) systems store energy in a rotating mass. Devices are composed of five main subsystems: flywheel, bearing, electrical machine, power converter and containment chamber. FES systems are not new but recent work has seen improvements in virtually every subsystem. In general, FES systems have shown extremely high efficiencies for short duration storage and have virtually unlimited temperature independent deep discharge capabilities [23]. This is topped with easy monitoring of state of charge. As seen in Table 3, adding to this list are the low environmental impacts, high specific power and power densities, good specific energy and energy densities, fast response times, very low capacity degradation, long life, high scalability, and the need for very little periodic maintenance [24]. Unfortunately, FES systems suffer from two main short comings: high self-discharge rates and safety [25].

Advances in materials have led to a transition from low speed (6000 rpm) steel flywheels to much higher speed, safer and more energy dense composite and alloy materials. Lower speed system costs can easily be five times less than higher speed equivalents however [23]. This is a result of the lower containment costs and lower cost of metals. Higher speed systems operate in the 10,000 to $110,000 \mathrm{rpm}$ regime $[23,26]$. This speed increase exploits the quadratic improvements resulting from higher speeds rather than those from higher mass. At these speeds, most of the flywheel capacity is stored in the higher velocity rotation and so flywheel speeds are rarely brought down below 50\% of their maximum [24,27]. The gains associated with the higher speeds have resulted in trends showing a shift to lower density and higher tensile strength materials. Among these materials are: aluminium alloys and graphite/carbon/glass fibre composites with added polymer (epoxy) resin support matrices [26,27]. Epoxy supported filament wound multi-ring flywheels have shown significant improvements in strength and safety [27,28]. Recent trends in carbon nanotubes have seen the emergence of biscrolling techniques to produce yarns for use in fibre composite flywheels [25]. These nanotubes could increase maximum speeds and subsequent storage densities quite significantly. However, increases in speed must be matched with improved and bulkier containment vessels which may eventually put an upper limit on storage capacities [27]. For safety, most system components are designed two times as strong as the maximum tensile strength of flywheel materials [29]. In portable applications, gimbal systems have been employed to counter gyroscopic precession [24].

Improvements in bearings are steadily reducing device self-discharge rates. Bearings usually take the form of one or more of the following combination: mechanical, fluid, diamagnetic, electromagnetic, 
superconducting and ferrofluid. Recent advances in materials have led to better quality mechanical bearings that are suitable for lower speed FES systems $(20,000$ to $40,000 \mathrm{rpm})$ [23,26]. These bearings have short life at the upper end of this low speed range [24]. As a slightly better replacement, fluid bearings may be used. At higher speeds frictional losses make mechanical and fluid bearings inappropriate. Instead electromagnetic and superconducting variants have been used. Permanent magnets are normally used to lift the flywheel mass. Constant energy input is then used to electromagnetically control and stabilize the levitated flywheel [24]. Mechanical bearings typically result in parasitic losses of about 5\% capacity or more per hour. This can be lowered to about $1 \%$ via electromagnetic bearings [29]. Electromagnetic variations suffer from iron losses related to stator and rotor eddy currents and material hysteresis. Recent advances in high temperature superconductors reduce rotor idling losses down to $0.1 \%$ of total capacity per hour [26]. Unfortunately, reducing the costs and size of cryogenic systems has proven difficult $[24,26]$. Typical FES development decouples design of bearing, electrical machine and flywheel subsystems. This approach is flexible but non-optimal. Recent efforts have attempted more holistic design methodologies. This new approach combined with carbon nanotubes and biscrolling techniques have led to the design of superconductor impregnated nanotube yarns [25]. These yarns could be used to produce a single part bearing and flywheel. Recently with the advent of stronger neodymium magnets and the improved availability of ultra-pure diamagnetic graphite the potential of diamagnetically stabilized magnetic bearings is becoming evident. These materials allow passive levitation and can reduce losses below the $0.1 \%$ of superconducting solutions [30]. The large scale feasibility and economic viability of diamagnetically stabilized bearings however remains in question. Brief investigations have also looked at ferrofluids as stabilizing materials for permanent magnet bearings but the viability of this approach also remains in question [31].

Permanent magnet (PM), induction machines and reluctance machines are the most common choice of electrical machines for FES [23]. The power dense PM machines have traditionally been chosen for high speed FES. These PM's eliminate copper losses in the rotor and so mitigate heat dissipation problems in the high vacuum environments of FES devices. They can, however, pose a problem not only in cost, but also with regards to demagnetization risk and low magnet tensile strength. Add to this cogging issues and associated idling losses and they quickly become less attractive [23,24]. Cogging issues can be reduced or eliminated with slotless stator designs at the expense of performance. This performance, in-turn can be improved through the use of halbach magnet configurations [32]. Recent advances in nano-materials have led to the development of high saturation exchange coupled "spring" magnets that increase flux densities and allow higher power output PM machines [25,33]. Asynchronous induction machines have also been proposed as rugged, low cost alternatives but complex rotor geometries, control methods and heat dissipation remain a significant issue. The simpler reluctance machines have gained more popularity as an alternative [34]. These machines, unfortunately, still have complex controls and are less energy dense than PM machines-recent improvements in power electronics have, however, simplified control. Issues such as torque ripple and acoustic noise that are prevalent in reluctance machines, fortunately remain less of a problem for flywheel systems than for other applications. Likewise, while heat dissipation issues also remain, they are less pronounced than those of induction machines [24]. Recently, hybrid PM reluctance machines attempt to take advantage of the positives of both individual machine types [35]. The added complexity of the flux path in these hybrid PM machines may explain why they have yet to gain popularity for FES systems. One additional 
point to note is that resistive losses (from copper windings) have been a significant concern for most machines. This has prompted efforts to change winding shapes and additional efforts to develop higher voltage machines [24]. These higher voltage machines have greater relevance for power plant scale applications. Finally, as opposed to traditional radial flux machines, axial flux machines have also been gaining greater interest for FES applications. These axial machines allow for easy cooling, planar adjustable air-gaps and increased power output (via the additional working surface).

Bi-directional power converters convert FES output into grid level voltages and generate variable speed control signals for energy storage [23]. The development of high power semiconductor switches has been critical to improvements in control. The low cost, compact, reliable and highly efficient design of GTO thyristors have traditionally made them the choice switch for power conversion. GTO's can only be triggered to on state (large off state current) and so the added convenience and higher switching frequencies of IGBTs have resulted in greater adoption for variable frequency power converters in recent years [24].

Most high speed FES systems operate under high vacuum to minimize the effects of friction. The high costs associated with pumping machinery and the poor heat dissipation characteristics have, however, prompted the use of low friction helium-air or other gas mixtures [26]. Safety is of primary importance for flywheel systems and a number of containment approaches have become increasingly common. Some of these apply directly to the design of the flywheels to produce high safety margin, non-bursting, incrementally failing flywheels. These types of flywheels reduce the requirements for containment from containing the full kinetic energy of the flywheel to simply retaining the failure within the chamber. Free floating containment vessels have been used to contain high energy, high impulse particulate fluid resulting from the disintegration of carbon-fibre composite flywheels operating at full speeds [36].

\subsection{Final Remarks}

Concerns of decreasing site availability, uncertain profitability, high capital costs, negative environmental impacts and low energy and power densities are increasingly suggesting a need to switch from PHS to another energy storage solution. CAES is showing greater energy and power densities and lower environmental impacts as well as greater site availability. Unfortunately, these improvements come at a fuel cost and commercial viability is yet to be tested. Flywheel systems, in comparison to CAES, are fairly mature and commercially tested. They exhibit many advantages over both PHS and CAES solutions. Safety issues associated with these systems can be mitigated but at a high cost. Both energy and power densities are, on average, higher than both PHS and CAES and show promise of even greater improvements with the advent of recent discoveries. But FES still operates on a relatively small scale and for very short durations. Whether or not improvements will be good enough to extend applicability to larger scale medium term solutions still remains to be seen. Because of their small scale these systems may be more relevant to deployment for distributed grid infrastructure. Flywheel systems have also seen applications both in power plant settings as uninterruptable power supplies and as hybrid renewable power smoothing solutions. 


\section{Chemical Energy Storage}

Chemical storage has by far the greatest diversity of research and commercial energy storage products to present day. Devices include not only traditional batteries, but also molten salt/liquid metal batteries, metal-air batteries, fuel cells and flow batteries.

\subsection{Typical Batteries}

The most known and widely used forms of chemical storage are our typical chemical batteries. Tables 4 and 5 along with Figures 5 and 6 examine and compare a select range of these batteries, namely: Zinc silver oxide ( $\mathrm{ZnAg}$ ), alkaline zinc manganese dioxide ( $\mathrm{ZnMn})$, lead acid ( $\mathrm{Pb}-\mathrm{Acid})$, lithium ion (Li-Ion), nickel metal hydride (NiMH), nickel cadmium ( $\mathrm{NiCd})$, nickel iron $(\mathrm{NiFe})$ and nickel zinc (NiZn) devices. These batteries consist of three main components: The positive (cathode) and negative (anode) electrodes, the electrolyte and the separator. Performance of traditional batteries is strongly linked to the material composition of the electrodes, with cycle life and lifespan being determined by the nature of the interface between electrolyte and electrode as well as electrode stability [37]. As a result, most chemistries are highly temperature sensitive and will experience a drop in capacity based on both environmental conditions and charge-discharge regimes. Likewise, rechargeable (secondary battery) chemistries tend to see a drop in performance when compared to primary batteries - this is usually a result of limited material availability for these configurations. Charge retention is usually measured in the range of years for primary batteries but drops to months in secondary battery systems. Many systems will experience pressure build-up and require venting. The difficulty of measuring state-of-charge for most batteries in this class can be added to the list of issues. Despite these issues, chemical batteries remain the best and most popular form of energy storage for smaller scale applications to present day.

Zinc silver oxide ( $\mathrm{ZnAg}$ ) batteries are known for their very high energy densities, low temperature performance, very flat discharge curves (under high and low current drain), low environmental impact and high durability. Unfortunately, the high cost of silver makes these batteries unsuitable for larger scale application and so uses have been restricted to hearing aids and watches [38] — though, some larger kilowatt scale banks exist. ZnAg batteries are generally single charge batteries with limited lifespans. For military applications, the need for long standby life has prompted mechanical and automatic means to active cells when needed, thus extending life- heaters are used to keep electrolyte temperatures up and improve activation [38]. The development of nano-materials has led to additional improvements in cathode design. Polymerization has resulted in improved layered separators. Recently implementations have seen the advent of low cycle life rechargeable $\mathrm{ZnAg}$ batteries for higher power mobile applications [39].

Alkaline zinc manganese dioxide ( $\mathrm{ZnMn}$ ) batteries are perhaps the most ubiquitous primary battery for portable devices in the market today. Unfortunately, this high demand has resulted in increased landfill waste and low recovery rates of Zinc, Manganese and Steel [40]. The popularity is a result of good low temperature and low/high current drain performance as well as low internal resistance. Discharge curves are not as flat as $\mathrm{ZnAg}$ chemistries, but costs are low. Long shelf life (shorter than $\mathrm{ZnAg}$ in un-activated state) makes $\mathrm{ZnMn}$ batteries suitable for uncontrolled storage in intermittent use applications. Newer batteries have greater resistance to internal pressure build-ups and resulting 
leakage [38]. Increasing power demands in portable electronics have pushed research into high drain ZnMn chemistries. The utilization of nano-materials has been a significant step in this direction [41]. Attempts to increase cycle life have resulted in bipolar rechargeable ZnMn devices [42]—with these new devices have come new charging regimes. Bismuth additions have also been used to extend this cycle life [43]. New advances in 3D printing have led to techniques for producing printable versions of ZnMn cells [44,45]. And finally, recent development efforts towards flow-assisted alkaline batteries have also looked at larger scale grid level applications [46].

Table 4. Comparing typical chemical batteries based on fourteen different metrics. Data obtained or calculated from multiple sources [7].

\begin{tabular}{|c|c|c|c|c|c|}
\hline \multirow{2}{*}{\multicolumn{2}{|c|}{ Metric }} & \multirow{2}{*}{$\begin{array}{c}\text { Zinc Silver Oxide } \\
\text { range } \sigma / \mu / \tilde{x} / n\end{array}$} & \multirow{2}{*}{$\begin{array}{c}\text { Alkaline } \\
\text { range } \sigma / \mu \sqrt[x]{x} n\end{array}$} & \multirow{2}{*}{$\begin{array}{c}\text { Lead Acid } \\
\text { range } \sigma / \mu \sqrt[x]{x} n\end{array}$} & \multirow{2}{*}{$\begin{array}{c}\text { Lithium Ion } \\
\text { range } \sigma / \mu / \widetilde{x} / n\end{array}$} \\
\hline & & & & & \\
\hline $\begin{array}{l}\text { Specific } \\
\text { Energy }\end{array}$ & {$[\mathrm{Wh} / \mathrm{Kg}]$} & $\begin{array}{c}81.00-276.00 \\
73.45 / 146.13 / 115 / 8\end{array}$ & $\begin{array}{c}80.0-175.00 \\
37.33 / 137 / 145 / 5\end{array}$ & $\begin{array}{c}10.00-50.00 \\
11.7 / 29.8 / 30.0 / 13\end{array}$ & $\begin{array}{c}30.00-300.00 \\
65.2 / 143.9 / 150 / 29\end{array}$ \\
\hline $\begin{array}{l}\text { Energy } \\
\text { Density }\end{array}$ & {$\left[\mathrm{KWh} / \mathrm{m}^{3}\right]$} & $\begin{array}{c}4.20-957.00 \\
322.93 / 308.51 / 179 / 8\end{array}$ & $\begin{array}{c}360.00-400.00 \\
22.54 / 386 / 398 / 3\end{array}$ & $\begin{array}{c}25.00-90.00 \\
19.7 / 59.6 / 55.0 / 11\end{array}$ & $\begin{array}{c}94.00-500.00 \\
137 / 290 / 250 / 17\end{array}$ \\
\hline $\begin{array}{c}\text { Specific } \\
\text { Power }\end{array}$ & {$[\mathrm{W} / \mathrm{Kg}]$} & $\begin{array}{c}0.09-330.00 \\
131.31 / 76.37 / 9.00 / 6\end{array}$ & $\begin{array}{c}4.35-35.0 \\
14.9 / 17.2 / 14.6 / 4\end{array}$ & $\begin{array}{c}25.00-415.00 \\
119.7 / 195 / 190 / 10\end{array}$ & $\begin{array}{c}8.00-2,000.00 \\
612.4 / 606 / 365 / 22\end{array}$ \\
\hline $\begin{array}{c}\text { Power } \\
\text { Density }\end{array}$ & {$\left[\mathrm{KW} / \mathrm{m}^{3}\right]$} & $\begin{array}{c}0.36-610.00 \\
243.14 / 151.88 / 28 / 6\end{array}$ & $\begin{array}{c}12.35-101.70 \\
43.7 / 49.3 / 41.6 / 4\end{array}$ & $\begin{array}{c}10.00-400.00 \\
185.8 / 123 / 41.9 / 4 \\
\end{array}$ & $\begin{array}{c}56.80-800.00 \\
321.4 / 366.4 / 304 / 4 \\
\end{array}$ \\
\hline Efficiency & {$[\%]$} & $\begin{array}{c}20.00-100.00 \\
34.25 / 68.75 / 77.50 / 4\end{array}$ & $\begin{array}{c}36.00-94.00 \\
25.5 / 73.0 / 81.0 / 4 \\
\end{array}$ & $\begin{array}{c}63.00-90.00 \\
8.56 / 76.9 / 76.0 / 15\end{array}$ & $\begin{array}{c}70.00-100.00 \\
8.5 / 89.8 / 90.0 / 17 \\
\end{array}$ \\
\hline Lifespan & {$[\mathrm{yr}]$} & $\begin{array}{c}2.00-10.00 \\
2.76 / 5.00 / 5.00 / 6\end{array}$ & $\begin{array}{c}2.50-10.00 \\
2.97 / 5.10 / 5.00 / 5\end{array}$ & $\begin{array}{c}3.00-20.00 \\
5.58 / 8.33 / 5.50 / 12\end{array}$ & $\begin{array}{c}2.00-20.00 \\
6.88 / 10.13 / 10.0 / 8\end{array}$ \\
\hline Cycle Life & [cycles] & $\begin{array}{c}1-1,500 \\
593.51 / 408.50 / 100 / 6\end{array}$ & $\begin{array}{c}1-200 \\
109.82 / 73.7 / 20 / 3 \\
\end{array}$ & $\begin{array}{c}100-2,000 \\
736 / 1,053 / 1,100 / 18\end{array}$ & $\begin{array}{c}250-10,000 \\
3,036 / 1,018 / 1,500 / 19\end{array}$ \\
\hline $\begin{array}{c}\text { Self- } \\
\text { Discharge } \\
\text { Rate } \\
\end{array}$ & {$[\% /$ day $]$} & $\begin{array}{c}0.01-0.25 \\
0.11 / 0.09 / 0.02 / 5\end{array}$ & $\begin{array}{c}0.008-0.011 \\
0.001 / 0.009 / 0.009 / 4\end{array}$ & $\begin{array}{c}0.033-1.10 \\
0.36 / 0.33 / 0.22 / 8\end{array}$ & $\begin{array}{c}0.03-0.33 \\
0.11 / 0.158 / 0.166 / 9\end{array}$ \\
\hline Scale & [MW] & $\begin{array}{c}0.00-0.25 \\
0.13 / 0.12 / 0.10 / 3\end{array}$ & $\begin{array}{c}0.00-0.001 \\
0.0005 / 0.0003 / 0 / 3\end{array}$ & $\begin{array}{c}0.00-50.00 \\
14.18 / 10.34 / 8 / 12 \\
\end{array}$ & $\begin{array}{c}0.00-3.00 \\
1.20 / 0.93 / 0.15 / 8\end{array}$ \\
\hline $\begin{array}{c}\text { Energy } \\
\text { Capital } \\
\text { Cost } \\
\end{array}$ & [US\$/KWh] & $\begin{array}{c}3,167.00-20,000.0 \\
6,732 / 9,795 / 6,686 / 7\end{array}$ & $\begin{array}{c}100.00-1,000.0 \\
407.9 / 463 / 283 / 5\end{array}$ & $\begin{array}{c}50.00-1,100.00 \\
231.4 / 303 / 250 / 24\end{array}$ & $\begin{array}{c}200.00-4,000.00 \\
1,076 / 1,110 / 600 / 15\end{array}$ \\
\hline $\begin{array}{c}\text { Power } \\
\text { Capital } \\
\text { Cost } \\
\end{array}$ & [US\$/KW] & $\begin{array}{c}7,140,620-741,935 \\
4,524 \mathrm{~K} / 3,941 \mathrm{~K} / \\
3,941 \mathrm{~K} / 2 \\
\end{array}$ & $\begin{array}{c}1,000-11,900 \\
5,994 / 5,008 / 2,125 / 3\end{array}$ & $\begin{array}{c}175.00-900.00 \\
209.1 / 383 / 300 / 15\end{array}$ & $\begin{array}{c}175.00-4,000.00 \\
1,446 / 2,325 / 1,950 / 9\end{array}$ \\
\hline App & cation & $\begin{array}{c}\text { Very Small Scale } \\
\text { Energy Management }\end{array}$ & $\begin{array}{c}\text { Very Small Scale } \\
\text { Energy Management }\end{array}$ & $\begin{array}{l}\text { Small/Medium Scale } \\
\text { Energy Management }\end{array}$ & $\begin{array}{l}\text { Small/Medium Scale } \\
\text { Energy Management }\end{array}$ \\
\hline Technic & Maturity & $\begin{array}{c}\text { Very Mature/Fully } \\
\text { Commercialized }\end{array}$ & $\begin{array}{c}\text { Very Mature/Fully } \\
\text { Commercialized }\end{array}$ & $\begin{array}{c}\text { Very Mature/Fully } \\
\text { Commercialized }\end{array}$ & $\begin{array}{c}\text { Mature/ } \\
\text { Commercialized }\end{array}$ \\
\hline Environm & tal Impact & Low & Medium & High & High/Medium \\
\hline
\end{tabular}


Table 5. Comparing typical chemical batteries based on fourteen different metrics.

Data obtained or calculated from multiple sources [7].

\begin{tabular}{|c|c|c|c|c|c|}
\hline \multicolumn{2}{|c|}{ Metric } & \multirow{2}{*}{$\begin{array}{c}\text { Nickel Metal } \\
\text { Hydride } \\
\text { range } \sigma / \mu / \tilde{x} / n\end{array}$} & \multirow{2}{*}{$\begin{array}{c}\text { Nickel Cadmium } \\
\text { range } \sigma / \mu / \tilde{x} / n \\
\end{array}$} & \multirow{2}{*}{$\begin{array}{l}\text { Nickel Iron } \\
\text { range } \sigma / \mu \widetilde{x} / n \\
\end{array}$} & \multirow{2}{*}{$\begin{array}{c}\text { Nickel Zinc } \\
\text { range } \sigma / \mu \widetilde{x} / n \\
\end{array}$} \\
\hline & & & & & \\
\hline $\begin{array}{l}\text { Specific } \\
\text { Energy }\end{array}$ & {$[\mathrm{Wh} / \mathrm{Kg}]$} & $\begin{array}{c}30.00-90.00 \\
20.2 / 66.5 / 73 / 14\end{array}$ & $\begin{array}{c}10.00-80.00 \\
20.5 / 45.3 / 45.0 / 16\end{array}$ & $\begin{array}{c}27.00-60.00 \\
14.3 / 43.1 / 45.0 / 7 \\
\end{array}$ & $\begin{array}{c}15.00-110.00 \\
29.7 / 69.6 / 72.5 / 12\end{array}$ \\
\hline $\begin{array}{l}\text { Energy } \\
\text { Density }\end{array}$ & {$\left[\mathrm{KWh} / \mathrm{m}^{3}\right]$} & $\begin{array}{c}38.90-300.00 \\
96.8 / 185 / 180 / 10 \\
\end{array}$ & $\begin{array}{c}15.00-150.00 \\
53.7 / 95.5 / 100 / 10 \\
\end{array}$ & $\begin{array}{c}25.00-80.00 \\
22.6 / 50.0 / 55.0 / 5 \\
\end{array}$ & $\begin{array}{c}80.00-400.00 \\
109.3 / 236 / 245 / 8 \\
\end{array}$ \\
\hline $\begin{array}{l}\text { Specific } \\
\text { Power }\end{array}$ & {$[\mathrm{W} / \mathrm{Kg}]$} & $\begin{array}{c}6.02-1,100.00 \\
378 / 408 / 235 / 12 \\
\end{array}$ & $\begin{array}{l}50.00-1,000.00 \\
244 / 222 / 150 / 13\end{array}$ & $\begin{array}{c}20.57-110.00 \\
45.8 / 54.9 / 25.0 / 5\end{array}$ & $\begin{array}{l}50.00-900.00 \\
287 / 201 / 95 / 8 \\
\end{array}$ \\
\hline $\begin{array}{c}\text { Power } \\
\text { Density }\end{array}$ & {$\left[\mathrm{KW} / \mathrm{m}^{3}\right]$} & $\begin{array}{c}7.80-588.00 \\
259.4 / 383 / 467 / 4 \\
\end{array}$ & $\begin{array}{c}37.66-141.05 \\
43.0 / 89.5 / 89.6 / 4 \\
\end{array}$ & $\begin{array}{c}12.68-35.18 \\
11.5 / 25.4 / 28.4 / 3 \\
\end{array}$ & $\begin{array}{c}121.38-608.00 \\
195.6 / 309 / 218 / 5 \\
\end{array}$ \\
\hline Efficiency & {$[\%]$} & $\begin{array}{c}50.00-80.00 \\
9.72 / 65.8 / 65.5 / 6 \\
\end{array}$ & $\begin{array}{c}59.00-90.00 \\
11.2 / 69.8 / 67.5 / 14 \\
\end{array}$ & $\begin{array}{c}65.00-80.00 \\
10.6 / 72.5 / 72.5 / 2 \\
\end{array}$ & $\begin{array}{c}80.00-89.00 \\
6.36 / 84.5 / 84.5 / 2 \\
\end{array}$ \\
\hline Lifespan & [yr] & $\begin{array}{c}2.00-15.00 \\
4.72 / 6.33 / 5.00 / 6 \\
\end{array}$ & $\begin{array}{c}2.00-20.0 \\
6.6 / 13.6 / 14.0 / 12 \\
\end{array}$ & $\begin{array}{c}8.00-100 \\
26.4 / 37.0 / 30.0 / 9 \\
\end{array}$ & $\begin{array}{c}1.00-10.00 \\
6.36 / 5.50 / 5.50 / 2 \\
\end{array}$ \\
\hline Cycle Life & [cycles] & $\begin{array}{c}300-3,000 \\
916 / 1,129 / 1,000 / 7\end{array}$ & $\begin{array}{c}300-10,000 \\
2,251 / 2,561 / 2,000 / 18 \\
\end{array}$ & $\begin{array}{c}1,000-8,500 \\
3,326 / 3,875 / 3,000 / 4 \\
\end{array}$ & $\begin{array}{c}100-500 \\
191.5 / 350 / 400 / 4 \\
\end{array}$ \\
\hline $\begin{array}{c}\text { Self- } \\
\text { Discharge } \\
\text { Rate } \\
\end{array}$ & [\%/day] & $\begin{array}{c}0.30-4.00 \\
1.27 / 1.16 / 0.83 / 7\end{array}$ & $\begin{array}{c}0.07-0.71 \\
0.23 / 0.34 / 0.25 / 12\end{array}$ & $\begin{array}{c}0.36-1.43 \\
0.39 / 0.80 / 0.71 / 6\end{array}$ & $\begin{array}{c}0.60-1.07 \\
0.25 / 0.79 / 0.71 / 3\end{array}$ \\
\hline Scale & {$[\mathrm{MW}]$} & $\begin{array}{c}0.01-3.00 \\
2.11 / 1.51 / 1.51 / 2 \\
\end{array}$ & $\begin{array}{c}0.00-50.00 \\
20.2 / 17.1 / 5.0 / 14 \\
\end{array}$ & $\begin{array}{c}0-0.05 \\
0.02 / 0.02 / 0.019 / 6 \\
\end{array}$ & $\begin{array}{c}0.001-0.05 \\
0.026 / 0.02 / 0.006 / 2 \\
\end{array}$ \\
\hline $\begin{array}{c}\text { Energy } \\
\text { Capital Cost } \\
\end{array}$ & [US\$/KWh] & $\begin{array}{c}200.00-729.00 \\
186.6 / 451 / 407 / 8 \\
\end{array}$ & $\begin{array}{c}330.00-3,500.0 \\
900 / 1,132 / 800 / 17\end{array}$ & $\begin{array}{c}444.27-1,316 \\
392 / 962 / 1,044 / 4 \\
\end{array}$ & $\begin{array}{c}250.00-660.00 \\
153.3 / 398 / 340 / 6 \\
\end{array}$ \\
\hline $\begin{array}{c}\text { Power } \\
\text { Capital Cost }\end{array}$ & [US\$/KW] & $\begin{array}{c}270.00-530.00 \\
145.7 / 362 / 286 / 3\end{array}$ & $\begin{array}{c}270.00-1,500.0 \\
494 / 867 / 600 / 9 \\
\end{array}$ & $\begin{array}{c}8,167-16,312 \\
4,107 / 12.5 \mathrm{~K} / 13.1 \mathrm{~K} / 1\end{array}$ & $\begin{array}{c}270.00-530.00 \\
183.9 / 400 / 400 / 2 \\
\end{array}$ \\
\hline Appli & ition & $\begin{array}{c}\text { Small Scale } \\
\text { Energy } \\
\text { Management }\end{array}$ & $\begin{array}{l}\text { Small/Medium Scale } \\
\text { Energy Management }\end{array}$ & $\begin{array}{l}\text { Small/Medium Scale } \\
\text { Energy Management }\end{array}$ & $\begin{array}{c}\text { Very Small Scale } \\
\text { Energy Management }\end{array}$ \\
\hline Technica & Maturity & $\begin{array}{c}\text { Very Mature/Fully } \\
\text { Commercialized }\end{array}$ & $\begin{array}{c}\text { Very Mature/Fully } \\
\text { Commercialized }\end{array}$ & $\begin{array}{c}\text { Mature/Limited } \\
\text { Development }\end{array}$ & $\begin{array}{c}\text { Mature/Limited } \\
\text { Development }\end{array}$ \\
\hline Environme & al Impact & High & High & Low & Low \\
\hline
\end{tabular}

Lead acid (Pb-Acid) batteries account for the largest secondary battery market share in the world. Despite lower performance than many existing chemical batteries, low cost combined with reasonable cycle life, wide operating temperatures, relatively high power outputs, relatively low maintenance and simple charging regimes make them attractive. They've seen applications in the SLI (starting, lighting, ignition) automotive industry as well as the telecom and uninterruptable power supply (UPS) industries. Some of the world's largest traditional chemical battery banks utilize these batteries. Valve regulated lead acid (VRLA) batteries have been critical for virtually maintenance free operation in these applications. Conical lead plate stacks have been used to produce round cell technologies for these low maintenance operations [38]. Sealed lead acid (SLA) batteries use gelled electrolytes and thick glass fibre absorbent mats to improve ruggedness. Despite some advantages, lead acid performance has been low and batteries can't be stored in discharged states due to sulfation effects that limit their lifespan [38]. They are also difficult to design in small packages and so have seen limited application in small portable 
electronics. Recently, load leveling applications involving Pb-Acid batteries used in conjunction with renewables have been explored. However, further improvements are still needed for these applications. Improved casing along with hybrid ultra-capacitor devices have aimed at improving performance by increasing specific power, recharge times and cycle life. Thin film variations, that lend themselves to low cost manufacture, have also seen recent entry into the consumer market. Titanium oxide substructures have been introduced to improve specific energy and produce bipolar batteries [47]. Finally, acid circulation can be used to enhance utilization of active materials. Despite improvements the nature of constituent materials means that these batteries continue to have high environmental impacts similar in levels to those of nickel cadmium batteries-fortunately, higher collection and recycling rates have mitigated some of these impacts [13].

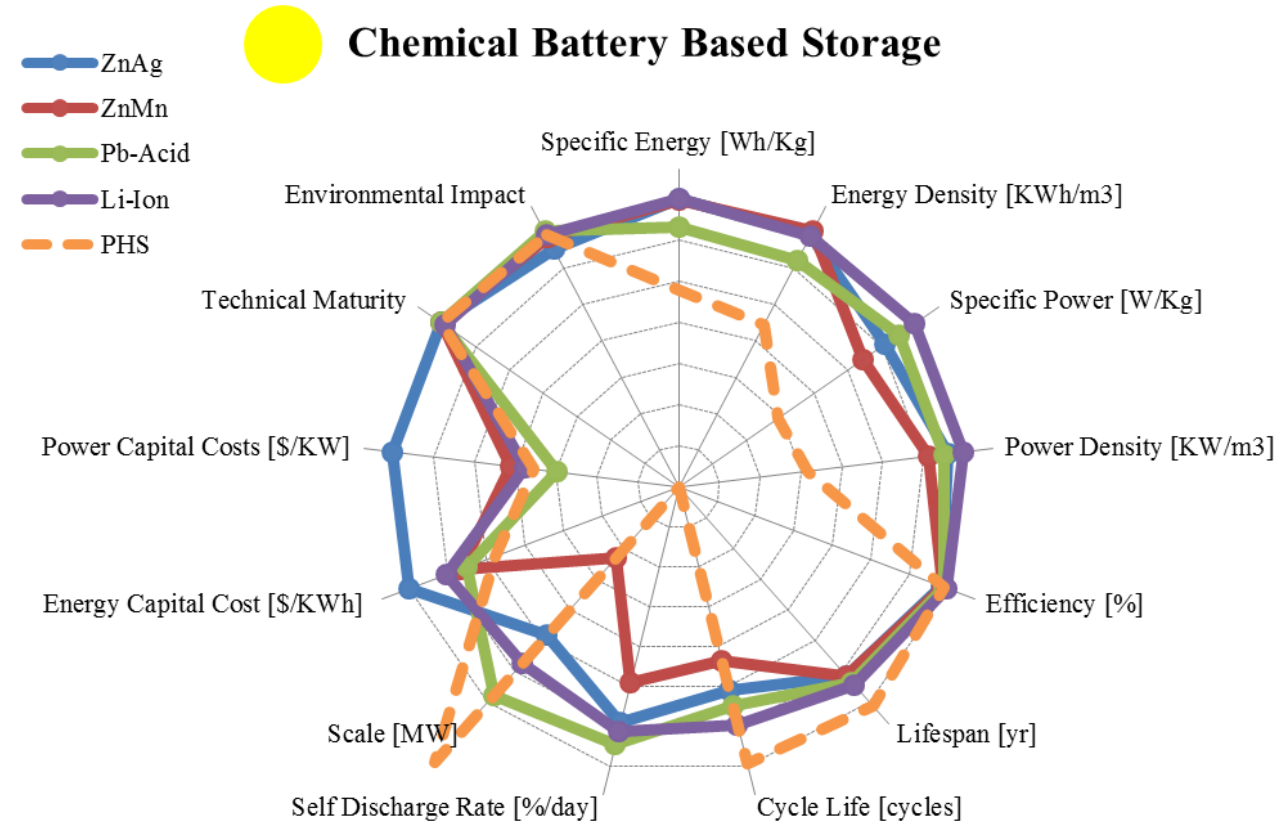

Figure 5. Comparing chemical energy storage in typically batteries using normalized and logarithmically plotted average data from Table 4.

Recent years have seen an increasing market for lithium ion (Li-Ion) batteries in portable electronics. Some high end chemistries have been used for space applications and a shift towards larger scale electric vehicle applications seems eminent. Li-Ion batteries have specific energy and energy densities close to those of alkaline primary batteries - this is higher than most existing rechargeable batteries. Additionally, Li-Ion devices have seen rapidly lowering costs, have excellent charge retention, high cell voltages, very good low temperature performance, long cycle life and high depth of discharge [38]. Unfortunately, there are some safety issues associated with these chemistries. Exceeding maximum charge voltages or physical device damage can lead to thermal runaway, venting, fire and explosion. Furthermore, pre-mature discharge cut-off or deep discharge can have permanent effects on battery capacity. This means that charging circuitry is complex and requires individual cell monitoring. Li-Ion devices are difficult to recycle [48] and concerns exist over the availability of sufficient resources for large scale storage applications [49]. Despite this they have lower environmental impacts than other battery types. Carbon fibre and nano-composite improvements in anode materials have aimed at higher power, greater reversibility and cycle life. Solid electrolytes and electrolyte salts have resulted in reduced 
acidity and greater stability to reduce safety risks. The use of layered cathodes along with the integration of internal electronics has also been proposed for newer battery designs [50]. Research has largely restricted itself to changes in electrolyte and negative electrode. These changes include the introduction of reversible intercalation, electrolyte polymerization (plastic lithium-ion thin film batteries), gel-coated micro-porous separators (lithium-ion polymer batteries) and much more. Further improvements involving the use of meso-porous materials and aerogels as well as the use of metal oxides in positive electrodes hold potential [37]. Despite these improvements, some have indicated that lithium chemistries are very much in their teenage years and much room for improvement exists [37].

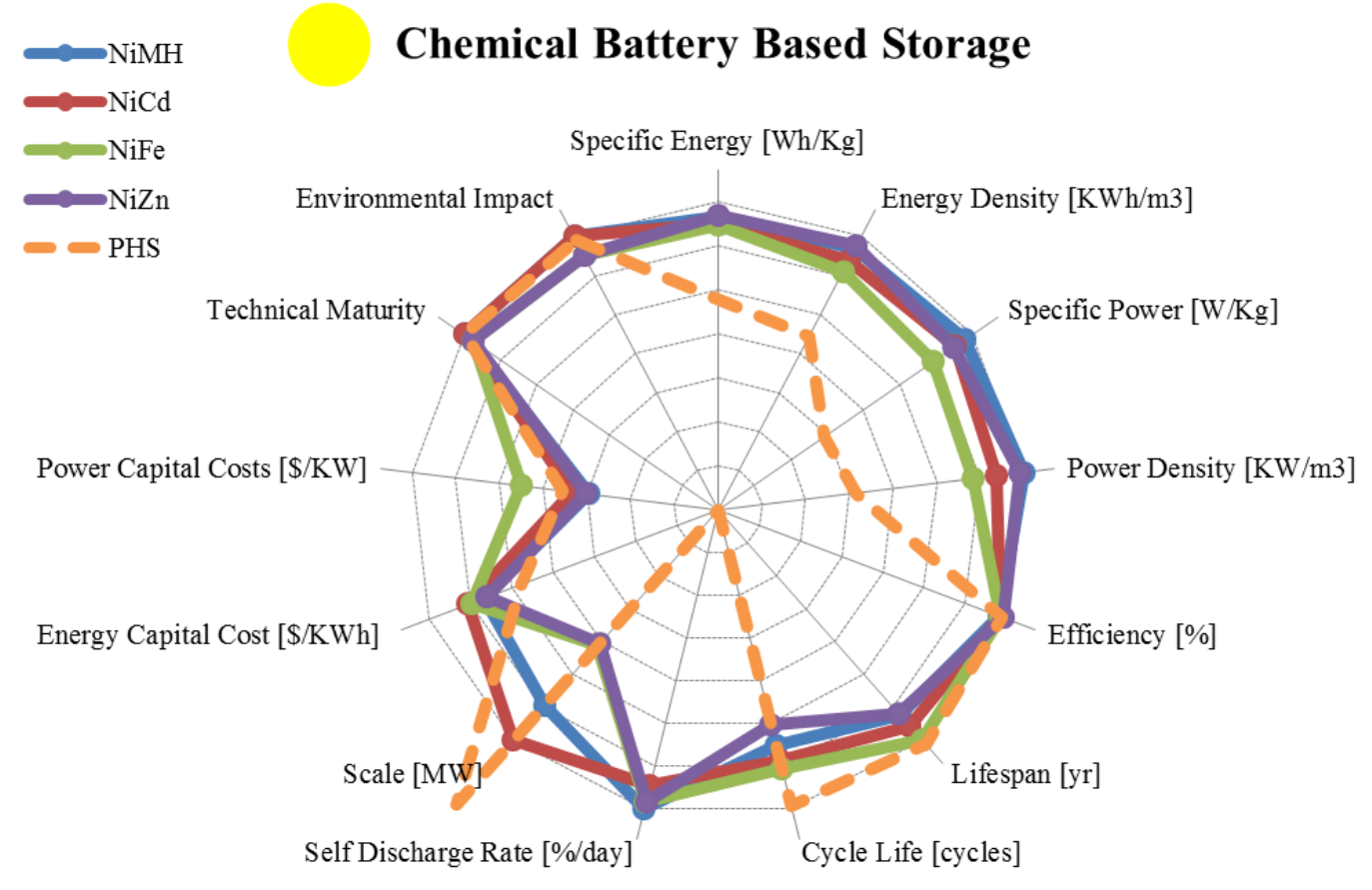

Figure 6. Comparing chemical energy storage in typically batteries using normalized and logarithmically plotted average data from Table 5.

Until recent Li-Ion improvements, nickel metal hydride (NiMH) batteries remained the choice devices for hybrid electric vehicles. They differ from existing chemistries in that they rely on reactions with hydrogen-ion absorbing porous metal alloys. NiMH systems are sealed, maintenance free, have low internal resistance and as seen in Tables 4 and 5, have higher energy densities than nickel cadmium chemistries, long shelf life (regardless of state of charge), long cycle life and rapid recharge capabilities. They also have higher costs and lower specific power than lithium ion batteries, moderate memory effect, limited operating temperature range, and only moderate charge retention. These negatives have resulted in a gradual displacement by Li-Ion cells. The relative abundance of materials has, however, made them more applicable for larger scale electric vehicle applications and to low power UPS systems [38]. Recent improvements have focused on improving specific power and low temperature performance [51]. Sintered plates have been used to increase porosity and charge retention [38]. Improvements in materials have aimed at changing electrode surface catalytic action at the metal-electrolyte interface [51]. Specialized activation processes have been suggested as methods of improving power performance [52]. The introduction of cobalt rich, zinc and manganese substitutions of nickel within the electrodes have improved temperature performance and also reduced corrosion issues, thus increasing cycle life $[51,52]$. 
If corrosion issues are successfully targeted, electrode particle size reduction has been noted as a method of increased power performance [52].

Nickel cadmium $(\mathrm{NiCd})$ batteries are very similar to $\mathrm{NiMH}$ chemistries but with cadmium replacing the metal alloy electrode. NiCd devices are very rugged and can withstand significant mechanical and electrical abuse. They are great for high power outputs, have good low temperature characteristics, wide temperature range, excellent cycle life, flat voltage profiles and are generally maintenance free [38]. The costs of nickel cadmium are equivalent to those of rechargeable alkaline batteries but remain higher than lead acid. NiCd batteries also exhibit memory effect when used for short periods of time at shallow discharge. Applications requiring moderate discharge are, therefore, preferred. Furthermore, cadmium is considered toxic and regulatory constraints have displaced these batteries in favor of NiMH and Li-Ion chemistries- $\mathrm{NiCd}$ is now restricted primarily to specialized applications, for instance, in aerospace. Most recent research has focused on reducing costs by more effectively using costly nickel and cadmium and simplifying manufacturing. In an attempt to increase power and energy densities, there has been a shift towards nickel foam, nickel fibre, plastic bonded and sintered electrodes - elasticity of fibre structured electrodes has resulted in reduced plate degradation [38].

Nickel iron (NiFe) batteries have the most rugged construction and the longest cycle life and highest durability of all batteries among traditional chemical batteries. They have the highest deep discharge capabilities and most flexible charging regimes as well making them the best chemistry for rough and rugged environments. These batteries were largely displaced with the advent of lead acids as a result of high manufacturing and maintenance costs, poor charge retention, high internal resistance and low energy and power densities. Recent developments have reduced costs and improved energy and power related performance. Costs of modern variations are higher than lead acids but lower than nickel cadmium batteries. Commercial applications in renewables power management are beginning to re-emerge. They have, also, once more begun to see applications in electric vehicles where cars have been known to run $150 \mathrm{Km}$ on a single charge [38]. Improvements have focused on the addition of sulfides, lithium and potassium additives, as well as on porous polymer separators and electro-precipitation techniques for efficient deposition of nickel into porous and metal fibre electrodes. Poor charge retention, heating issues during charge/discharge and some minor hydrogen venting issues remain the primary difficulty with NiFe devices [53].

Nickel zinc (NiZn) batteries have higher specific energy than other nickel chemistries. They have seen primary application in small electric scooters and bicycles. They have high rate capabilities, can be discharged completely and charged rapidly, are sealed and maintenance free, have low environmental impact and are made with widely available materials [38]. They are, however, of higher cost than lead acid batteries and have low energy densities. The single biggest hurdle to adoption has been their short cycle life. Recent efforts have focused on improving this cycle life via one of five routes: Electrode additives, electrolyte additives, improved separators, changes in charging regimes or changes in active material synthesis. Addition of mercury and other heavy metals as well as hydroxides has improved zinc electrode stability and reduced loss of active material. Polymer binding has reduced shape change effects to reduce zinc loss (and capacity loss) even further. Reduced alkalinity of electrolytes have done the same. PVA film separators have added flexibility without wetting expansion to reduce shape change effects as well. Pulsed DC charging regimes have been used to extend life and the use of foams and 
nano-materials for electrodes have improved base capacities. Despite all these improvements NiZn still have unsatisfactory cycle life [53].

\subsection{Liquid Metal, Molten Salt and Metal Air Batteries}

Outside of our traditional chemical batteries, recent years have seen the rise of newer approaches. These have included the use of high temperature batteries that utilize molten salts and liquid metals. Two molten salt batteries: Sodium sulphur $(\mathrm{NaS})$ and sodium nickel chloride $(\mathrm{NaNiCl})$ are considered for this review (see Table 6 and Figure 7) - these batteries utilize liquid/molten salts as electrolytes which also play the part of electrodes - the electrodes are separated by a solid membrane separator. Recent research has also seen the advent of liquid metal variations that utilize liquid metal electrodes as well as molten salt electrolytes [54] — these batteries are at the present in very early stage development and have yet to see any viable commercial applications. Both molten salt and liquid metal batteries have aimed at targeting the larger grid scale energy storage applications not normally accessible to traditional battery chemistries. Working examples of molten salt systems exist in Japan as well as the United States [55,56]. Thermal management for these battery types is critical and operation should be continuous to prevent a transition from liquid to solid phase. Moving away from molten battery chemistries, other interesting changes and novelties have included batteries that replace the second electrode with an air electrode - these batteries operate on hydroxyl ion exchange to produce energy. These metal-air chemistries may hold promise for grid scale applications as well. Two batteries: Zinc-air (Zn-Air) and iron-air (Fe-Air) are considered for this review (see Table 6).

Sodium sulfur $(\mathrm{NaS})$ batteries have primarily been aimed at utility scale storage for load leveling applications $[55,56]$. Some uses for electric vehicles also exist. These devices are proven and mature but have seen limited but rising commercial application. NaS batteries have the potential for lower costs than traditional chemical batteries. They operate at high temperatures of around $300-350{ }^{\circ} \mathrm{C}$. They have high energy density and good power density, are efficient if continuously used and operate independent of external temperature variations. Flexible operation with little maintenance and very good cycle life combined with easy state of charge monitoring and low (close to zero) self-discharge rates have made these batteries very attractive. Furthermore, the raw materials for production of these batteries are abundant and inexpensive. Molten reactants eliminate issues of mechanical wear and other aging effects, but mean that thermal management is required [38]. Thermal management is usually easy but generally requires several systems: Heating systems, insulation systems and cooling systems.

In $\mathrm{NaS}$ chemistries, heating raises and maintains the battery temperature at the operating range. Some of this heating can occur during cell discharge, so maximum efficiency is obtained through continuous discharge regimes [56]. Mechanisms for even heat distribution are also needed. This heating requirement is a major source of loss and requires constant grid connection after activation. Thermal properties have, thus, been the focus of research [57]. Insulation can help reduce this loss. For large utility scale applications insulation can take the form of conventional fibre board or micro-porous materials - these materials are cheaper and existing grid infrastructure can be used to provide energy for re-injecting lost heat. Low density active materials reduce structural requirements. For portable applications higher quality and more expensive but less bulky evacuated insulation is required. The additional use of variable conductance insulation allows electronically controllable metal hydride hydrogen absorption for better 
thermal management $[58,59]$. Heat dissipation and cooling is directly linked to efficiency and in addition to variable conductance approaches, may be done through heat pipes, thermal shunts, latent heat storage, evaporative cooling and air/liquid heat exchange [38].

Table 6. Comparing molten salt and metal-air chemical batteries based on fourteen different metrics. Data obtained or calculated from multiple sources [7].

\begin{tabular}{|c|c|c|c|c|c|}
\hline \multirow{2}{*}{\multicolumn{2}{|c|}{ Metric }} & Sodium Sulphur & Sodium Nickel Chloride & Zinc Air & Iron Air \\
\hline & & $\operatorname{range} \sigma / \mu / \tilde{x} / n$ & $\operatorname{range} \sigma / \mu / \tilde{x} / n$ & $\operatorname{range} \sigma / \mu / \tilde{x} / n$ & $\operatorname{range} \sigma / \mu / \widetilde{x} / n$ \\
\hline $\begin{array}{l}\text { Specific } \\
\text { Energy }\end{array}$ & {$[\mathrm{Wh} / \mathrm{Kg}]$} & $\begin{array}{c}100.00-240.00 \\
44.72 / 145 / 135 / 10\end{array}$ & $\begin{array}{c}85.00-140.00 \\
16.7 / 112 / 117 / 10\end{array}$ & $\begin{array}{c}10.00-470.00 \\
165 / 227 / 150 / 15\end{array}$ & $\begin{array}{c}8.00-109.00 \\
28.9 / 68.1 / 70.0 / 9\end{array}$ \\
\hline $\begin{array}{l}\text { Energy } \\
\text { Density }\end{array}$ & {$\left[\mathrm{KWh} / \mathrm{m}^{3}\right]$} & $\begin{array}{c}150.00-345.00 \\
62.8 / 213 / 190 / 10 \\
\end{array}$ & $\begin{array}{c}108.00-190.00 \\
30.6 / 159.5 / 165 / 6 \\
\end{array}$ & $\begin{array}{c}22.00-1,673.00 \\
791 / 816.5 / 786 / 6 \\
\end{array}$ & $\begin{array}{l}100.00-1,000.0 \\
636.4 / 550 / 550 / 2 \\
\end{array}$ \\
\hline $\begin{array}{l}\text { Specific } \\
\text { Power }\end{array}$ & {$[\mathrm{W} / \mathrm{Kg}]$} & $\begin{array}{c}14.29-260.00 \\
86.5 / 176.0 / 230 / 9 \\
\end{array}$ & $\begin{array}{c}10.00-260.00 \\
76.5 / 143 / 150 / 9 \\
\end{array}$ & $\begin{array}{c}60.00-225.00 \\
56.1 / 117 / 100 / 9 \\
\end{array}$ & $\begin{array}{c}18.86-146.00 \\
54.7 / 81.7 / 81.0 / 4 \\
\end{array}$ \\
\hline $\begin{array}{l}\text { Power } \\
\text { Density }\end{array}$ & {$\left[\mathrm{KW} / \mathrm{m}^{3}\right]$} & $\begin{array}{c}1.33-50.00 \\
17.6 / 21.8 / 20.0 / 5\end{array}$ & $\begin{array}{c}54.20-300.00 \\
96.5 / 219.2 / 257 / 5\end{array}$ & $\begin{array}{c}10.00-208.00 \\
91.5 / 74.9 / 40.9 / 4\end{array}$ & $\begin{array}{c}250 \\
\mathrm{x} / 250 / 250 / 1\end{array}$ \\
\hline Efficiency & [\%] & $\begin{array}{c}65.00-92.00 \\
8.1 / 81.5 / 85.0 / 21 \\
\end{array}$ & $\begin{array}{c}21.00-92.50 \\
34.2 / 72.1 / 87.5 / 4\end{array}$ & $\begin{array}{c}30.00-50.00 \\
9.60 / 44.25 / 48.5 / 4\end{array}$ & $\begin{array}{c}42.00-96.00 \\
20.2 / 66.5 / 62.5 / 8\end{array}$ \\
\hline Lifespan & {$[\mathrm{yr}]$} & $\begin{array}{c}5.00-20.0 \\
5.07 / 12.22 / 15.0 / 9\end{array}$ & $\begin{array}{c}7.00-14.00 \\
3.10 / 9.75 / 9.00 / 4\end{array}$ & $\begin{array}{c}0.17-30.00 \\
12.6 / 8.08 / 3.00 / 5\end{array}$ & Long \\
\hline Cycle Life & [cycles] & $\begin{array}{c}1,000-4,500 \\
1,222 / 2,771 / 2,500 / 12\end{array}$ & $\begin{array}{c}2,000-3,000 \\
500 / 2,500 / 2,500 / 3\end{array}$ & $\begin{array}{c}1.00-500.00 \\
251.2 / 234 / 200 / 3\end{array}$ & $\begin{array}{c}100-5,000 \\
1,580 / 1,089 / 400 / 9\end{array}$ \\
\hline $\begin{array}{c}\text { Self- } \\
\text { Discharge } \\
\text { Rate } \\
\end{array}$ & {$[\% /$ day $]$} & $\begin{array}{c}0.00-20.0 \\
10.95 / 8.01 / 0.05 / 5\end{array}$ & $\begin{array}{c}11.89-26.25 \\
7.56 / 17.7 / 15.0 / 3\end{array}$ & $\begin{array}{c}0.005-0.01 \\
0.003 / 0.007 / 0.005 / 3\end{array}$ & Small \\
\hline Scale & [MW] & $\begin{array}{c}0.01-80.00 \\
22.6 / 13.1 / 4.5 / 16\end{array}$ & $\begin{array}{c}0.00-53.00 \\
21.5 / 12.3 / 0.30 / 7\end{array}$ & $\begin{array}{c}0.00-1.00 \\
0.41 / 0.17 / 0.008 / 6\end{array}$ & $\begin{array}{c}0.00-0.01 \\
0.005 / 0.006 / 0.006 / 5\end{array}$ \\
\hline $\begin{array}{c}\text { Energy } \\
\text { Capital } \\
\text { Cost } \\
\end{array}$ & {$[\mathrm{US} \$ / \mathrm{KWh}]$} & $\begin{array}{c}150.00-900.00 \\
177.6 / 387 / 350 / 14\end{array}$ & $\begin{array}{c}100.00-345.00 \\
100.9 / 211 / 200 / 4\end{array}$ & $\begin{array}{c}10.00-950.00 \\
378 / 313 / 130 / 6\end{array}$ & $\begin{array}{c}10.00-150.00 \\
50.2 / 70.0 / 65.0 / 6\end{array}$ \\
\hline $\begin{array}{c}\text { Power } \\
\text { Capital } \\
\text { Cost } \\
\end{array}$ & {$[\mathrm{US} \$ / \mathrm{KW}]$} & $\begin{array}{c}150.00-3,300.0 \\
1,121 / 1,736 / 1,850 / 13\end{array}$ & $\begin{array}{c}150.00-10,000 \\
4,615 / 3,613 / 2,150 / 4\end{array}$ & $\begin{array}{c}100.00-4,000.0 \\
1,567 / 1,533 / 975 / 6\end{array}$ & $\begin{array}{c}950 \\
\mathrm{x} / 950 / 950 / 1\end{array}$ \\
\hline \multicolumn{2}{|c|}{ Application } & $\begin{array}{l}\text { Medium/Large Scale } \\
\text { Energy Management }\end{array}$ & $\begin{array}{l}\text { Medium/Large Scale } \\
\text { Energy Management }\end{array}$ & $\begin{array}{c}\text { Very Small Scale } \\
\text { Energy Management }\end{array}$ & $\begin{array}{c}\text { Small Scale } \\
\text { Energy Management }\end{array}$ \\
\hline \multicolumn{2}{|c|}{ Technical Maturity } & $\begin{array}{c}\text { Proven/ } \\
\text { Commercializing }\end{array}$ & $\begin{array}{c}\text { Proven/ } \\
\text { Commercializing }\end{array}$ & $\begin{array}{c}\text { Mature/ } \\
\text { Commercialized }\end{array}$ & $\begin{array}{l}\text { Research/ } \\
\text { Developing }\end{array}$ \\
\hline Environm & tal Impact & Medium/Low & Medium/Low & Very Low & Very Low \\
\hline
\end{tabular}




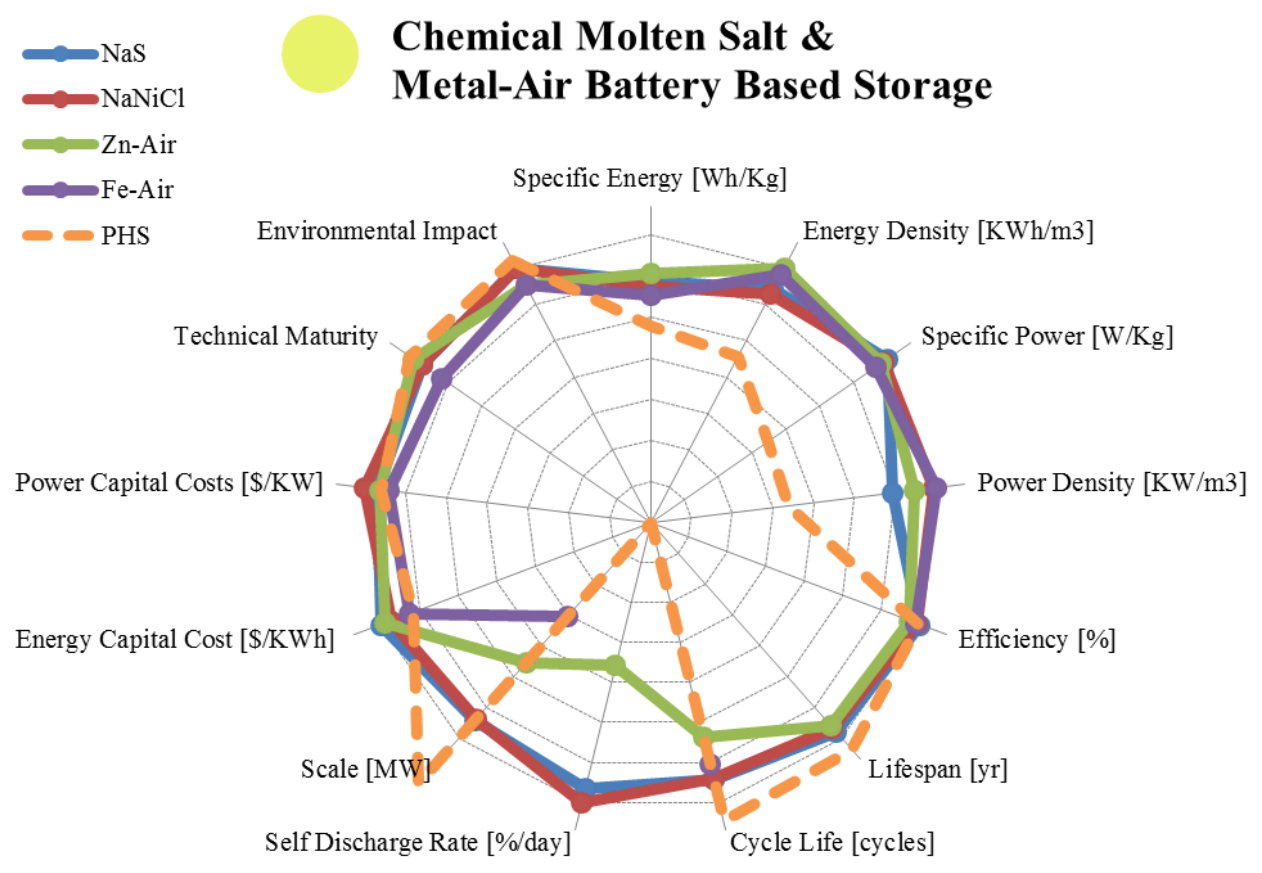

Figure 7. Comparing chemical energy storage in molten salt and metal-air batteries using normalized and logarithmically plotted average data from Table 6.

Safety issues exist in NaS systems. These are primarily related to the corrosive nature of molten salts and their appropriate disposal. The use of properly designed enclosures have mitigated most environmental hazards and have made these batteries are relatively benign [38] - these enclosures use chromium and molybdenum-lined vessels [60]. Failure of molten salt separator barriers occurs in a fail-safe manner and results in sodium-sulfur reactions to produce solid barriers that cut-off cell operation. Furthermore, seal failure can be mitigated by redirecting molten salt flows. Recent refinements have looked at tubular designs that minimize electrode volume and allow for reduced sealing areas [61]. The corrosive nature of high temperature salts has led to a shift towards lower temperature $\left(<100{ }^{\circ} \mathrm{C}\right)$ implementations - these have conventional separators and, while theoretically higher capacity, have suffered from intermediate polysulfide reactions with electrodes [60]. Protective electrode layers have thus become a key focus of these lower temperature chemistries.

Additional refinements have looked at pulsed power generation to provide up to $500 \%$ over capacity for short duration power quality applications $[38,56]$. Other improvements have attempted to increase energy and power performance. Focus on a range of fast sodium ion conductors (including beta-alumina ceramics) for separator material has been an important step in this direction [60,61]. To improve capacity even further the use of layered oxides has been explored [60]. NaS applications have also been expanded to hybrid wind and fuel cell electricity storage [61]. Despite this, devices remain somewhat expensivethis expense has proven to be a barrier to commercialization.

Both the sodium sulfur $(\mathrm{NaS})$ and the sodium nickel chloride $(\mathrm{NaNiCl})$ chemistries share a number of common traits. $\mathrm{NaNiCl}$ batteries, unlike $\mathrm{NaS}$, were designed primarily for the electric vehicle industry. They have been far more robustly tested for mobile regimes and undergone crash tests, over charge tests, water immersion, fire exposure and destructive shorting tests [38]. Batteries are robust to failure, have passed these tests and shown continued operation in some cases. These batteries also contain less corrosive materials and operate at lower temperatures $\left(270{ }^{\circ} \mathrm{C}\right)$ than $\mathrm{NaS}$ batteries- 
consequently they have shown lower environmental risks. Assembly in the discharged state is also possible, thus mitigating some safety concerns during manufacture [60]. One popular commercial $\mathrm{NaNiCl}$ battery type is called the ZEBRA battery [62]. Developments of ZEBRA batteries have shown that passive means are very effective cooling measures for these battery systems [56,62]. Recent work on iron additions have shown increases in power response making these batteries more competitive than $\mathrm{NaS}$ variations in this respect [60]. Ultimately, both $\mathrm{NaS}$ and $\mathrm{NaNiCl}$ batteries are poised for a come-back, especially given that they are one of the few viable large scale storage solutions and given that traditional lithium chemistries have had a number of sustainability issues. Future improvements can potentially make them price competitive with pumped hydro and compressed air storage.

At this point, we take a slight departure from the molten salt regime to look at metal-air batteries. While a number of chemistries exist, the next few paragraphs will focus on zinc-air (Zn-Air) and iron-air (Fe-Air) batteries. These batteries are a cross-over of traditional chemical batteries and fuel cells. Most metal-air batteries have a number of common features. They tend to have one anode electrode (aluminium, zinc, iron, lithium are the most common). The second electrode is an oxygen electrode that catalyzes the production of hydroxyl ions - a separator is designed to allow ion flow and prevent flow (and loss) of the anode electrode material. As seen from Table 6, these chemistries offer very high energy densities in comparison to most chemical batteries - in-fact, they offer the highest energy densities of most storage systems in general. Out of the metal-air chemistries, lithium chemistries are so energy dense that they are comparable to some hydrocarbon fuels. But most batteries suffer from extremely poor cycle and shelf life as a result of hydrogen evolution [38]. Binding and gelling agents have recently been used to reduce hydrogen evolution [63]. These batteries are usually suitable for low discharge rates with some high rate short duration pulse application - this application is possible as a result of oxygen buildup in the cell. Incorporation of an air reservoir has been proposed as a method of improving pulse performance [38]. Most metal-air configurations are also highly prone to negative performance effects resulting from environmental changes - increases or decreases in water content can change electrolyte concentration to non-optimal values. Normally these batteries are primary batteries that require mechanical recharge. However, recent work has focused on bi-functional oxygen electrodes that can allow electric chargeability [38].

Zinc air (Zn-Air) variations tend to have relatively flat discharge voltages on low current draws. Unlike lithium-air versions, they are cheap, environmentally benign and have long storage life while un-activated [38]. However, like other metal-air chemistries, Zn-Air batteries are highly sensitive to changes in temperature, humidity and other factors. The high energy densities have resulted in potential electric vehicles applications whereby mechanical recharge is done via replaceable anode cassettes $[64,65]$. To improve high rate performance $\mathrm{Zn}$-Air batteries have sometimes been hybridized with nickel cadmium or manganese dioxide chemistries [38]. Even without hybridization, recent power capacity improvements have made Zn-Air batteries applicable to hearing-aids and other small electronics. In attempts to make further improvements, improved air flow has been considered at the expense of shortened life. Shelf life has been extended by storing cells in un-activated conditions and activating via physical means or, in the case of larger devices, via water activation. The shortened life is a result of corrosion of zinc as well as gas transfer problems. Gas intake problems can be reduced by blocking air-intake and extending shelf life. Improvements in stability of zinc electrodes have focused on nickel alloying and addition of bismuth, while gelling and binding techniques have increased lifespan 
even further. Electrolyte additives such as PEG have inhibited hydrogen evolution to reduce corrosion effects. Inorganic materials introduced into separator micro-pores have also reduced active material losses and increased lifespan [63]. Electrode development has largely been limited to intercalation chemistries. These chemistries allow improved porosities and hence improved capacities. Recent developments have also resulted in the production of high capacity thin fibre substrated laminated air electrodes [38]. Hydrophobicity, combined with gas porosity and Teflon bonded catalyst structures hold the potential for high performance air/gas electrodes [38]. Despite these improvements, Zn-Air batteries still suffer from significant high rate capacity and cycle life issues and so remain confined to specialized small scale applications.

Recent improvements applied to iron-air (Fe-Air) batteries, however, may have led to breakthroughs in large scale energy storage. These batteries replace zinc with iron. Unlike zinc, iron doesn't suffer from severe active material redistribution and resulting shape changes - this prolongs life. Furthermore hydroxides of iron have low solubility in the alkaline electrolyte and so corrosion effects remain low. Like Zn-Air though, high discharge capabilities remain poor and charging efficiency remains low. Recent developments involving the use of ultra-pure magnetite and carbonyl iron, combined with bismuth sulfide additions and additional coating materials have resulted in significant improvements [66]. Cycle life improvements of up to 5000 cycles and efficiency improvements of up to $80 \%$ may now be possible. Combined with the low cost of iron (per KWh of energy) means that very competitive large scale energy storage via Fe-Air batteries may be just around the corner. Unfortunately, Fe-Air batteries are still very new and data associated with the technology is sparsely available.

\subsection{Fuel Cells}

Fuel cells and metal-air batteries share many similar traits. Fuel cells have seen extensive development as alternative storage and generation technologies. It should be noted that fuel cells are more appropriately classified as generation devices, not storage devices. Unlike other chemical batteries, they receive an inflow of fuel (or active material) from an external source and convert it into electrical output. The fuel is oxidized at the anode and reduced at the cathode. This conversion can happen directly from the fuel (i.e., hydrogen, methanol, hydrazine) or indirectly via an intermediate pre-processing step (reforming) to convert the fuel (i.e., natural gas, ammonia, ethanol, hydrocarbon gases) into a hydrogen rich gas. Fuel cells are composed of two electrodes with an electrolyte sandwiched between them - a catalyst is interleaved between the electrolyte-electrode interface layers. These single electrode-electrolyte-catalyst assembles are stacked to give higher voltages. Additional fuel and waste management (via flow control, storage) and power system conversion must occur for complete cell stacks [38]. Complete systems are sometimes regenerative and can combine electrolyzer and waste processing for closed loop operation. Unlike typical heat engines, these devices are not limited by Carnot cycle efficiencies and so hold potential as cleaner, quieter and higher efficiency alternatives to typical generation. Most existing fuel cell technologies are in the very early stages, with some limited application-specific implementations in aerospace or large scale grid backup generation.

Key problems with fuel cell deployment on a commercial scale appear to be those of lifespan and cost. High costs and lifespan are partially a result of platinum group noble metal catalysts and their degradation. As we see from Table 7 and Figure 8, however, these devices have high specific power and 
energy as well as high energy densities - they do considerably better than existing chemical batteries (with the exception of metal-air chemistries) but power densities remain low. Trace impurities in fuel source can also result in significant performance drops and have been an area of active research. While fuel cells have traditionally been regarded as clean technologies, gaseous fuel loss combined with impurities and resultant corrosion gases can have significant long term negative impact on atmosphere and ecology [67]. Some particularly interesting work associated with greener energy production and storage relates to the use of microbial or enzymatic fuel cells to produce energy from biological action and more benign fuels such as sugar [43]. In general, the goal of recent research has focused on bringing fuel cells to either portable applications or up-to grid level applications at lower costs - both these applications will require significant innovation. This section looks at four particular fuel cell technologies, namely: Proton Exchange Membrane (PEMFC), Direct Methanol (DMFC), Molten Carbonate (MCFC) and Solid Oxide (SOFC). Of-course, a wide array of other fuel cell technologies also exist, but are not addressed here.

Table 7. Comparing fuel cells (chemical batteries) based on twelve different metrics. Data obtained or calculated from multiple sources [7].

\begin{tabular}{|c|c|c|c|c|c|}
\hline \multicolumn{2}{|c|}{ Metric } & $\begin{array}{c}\text { Polymer Exchange } \\
\text { Mem. }\end{array}$ & Direct Methanol & Molten Carbonate & Solid Oxide \\
\hline $\begin{array}{l}\text { Specific } \\
\text { Energy }\end{array}$ & {$[\mathrm{Wh} / \mathrm{Kg}]$} & $\begin{array}{c}100.00-450.00 \\
135 / 246.6 / 200 / 13\end{array}$ & $\begin{array}{c}140.30-960.00 \\
308.4 / 400 / 300 / 9\end{array}$ & $\begin{array}{c}369.00-607.00 \\
168.3 / 488 / 488 / 2 \\
\end{array}$ & $\begin{array}{c}410.00-1,520.0 \\
513 / 773 / 582 / 4 \\
\end{array}$ \\
\hline $\begin{array}{l}\text { Energy } \\
\text { Density } \\
\end{array}$ & {$\left[\mathrm{KWh} / \mathrm{m}^{3}\right]$} & $\begin{array}{c}112.20-770.00 \\
211.7 / 380.2 / 360 / 8 \\
\end{array}$ & $\begin{array}{c}29.90-274.00 \\
90.08 / 135 / 118 / 6 \\
\end{array}$ & $\begin{array}{c}25.00-40.00 \\
10.6 / 32.5 / 32.5 / 2 \\
\end{array}$ & $\begin{array}{c}172.00-462.09 \\
160.5 / 277 / 198 / 3 \\
\end{array}$ \\
\hline $\begin{array}{c}\text { Specific } \\
\text { Power }\end{array}$ & {$[\mathrm{W} / \mathrm{Kg}]$} & $\begin{array}{c}4.00-150.00 \\
63.7 / 56.1 / 18.2 / 5 \\
\end{array}$ & $\begin{array}{c}2.10-20.00 \\
5.53 / 11.1 / 10.0 / 9\end{array}$ & $\begin{array}{c}12.00-36.70 \\
11.1 / 22.4 / 20.4 / 4\end{array}$ & $\begin{array}{c}10.00-63.34 \\
24.9 / 27.3 / 12.8 / 6 \\
\end{array}$ \\
\hline $\begin{array}{l}\text { Power } \\
\text { Density }\end{array}$ & {$\left[\mathrm{KW} / \mathrm{m}^{3}\right]$} & $\begin{array}{c}4.20-35.00 \\
15.48 / 18.7 / 16.9 / 3 \\
\end{array}$ & $\begin{array}{c}1.00-300.00 \\
103.6 / 44.1 / 6.9 / 8 \\
\end{array}$ & $\begin{array}{c}1.05-1.67 \\
0.44 / 1.36 / 1.36 / 2\end{array}$ & $\begin{array}{c}4.20-19.25 \\
8.61 / 9.31 / 4.47 / 3\end{array}$ \\
\hline Efficiency & {$[\%]$} & $\begin{array}{c}22.00-85.00 \\
15.21 / 46.36 / 40 / 25\end{array}$ & $\begin{array}{c}10.00-40.00 \\
10.3 / 23.3 / 20.0 / 6 \\
\end{array}$ & $\begin{array}{c}45.00-80.00 \\
10.4 / 55.4 / 52.5 / 10 \\
\end{array}$ & $\begin{array}{c}50.00-65.00 \\
4.76 / 58.6 / 60.0 / 7\end{array}$ \\
\hline Lifespan & [yr] & $\begin{array}{c}0.22-10.00 \\
4.04 / 4.07 / 2.79 / 10\end{array}$ & $\begin{array}{c}0.01-0.56 \\
0.22 / 0.24 / 0.22 / 5\end{array}$ & $\begin{array}{c}1.40-10.00 \\
3.14 / 4.90 / 4.50 / 5\end{array}$ & $\begin{array}{c}0.28-10.00 \\
5.09 / 4.26 / 2.50 / 3\end{array}$ \\
\hline Scale & {$[\mathrm{MW}]$} & $\begin{array}{c}0.00-50.00 \\
17.58 / 6.49 / 0.18 / 8\end{array}$ & $\begin{array}{c}0.00-1.00 \\
0.37 / 0.16 / 0.001 / 7\end{array}$ & $\begin{array}{c}0.01-100.00 \\
48.6 / 39.2 / 2.0 / 7\end{array}$ & $\begin{array}{c}0.00-100.00 \\
40.7 / 17.0 / 0.10 / 6 \\
\end{array}$ \\
\hline $\begin{array}{c}\text { Energy } \\
\text { Capital Cost } \\
\end{array}$ & [US\$/KWh] & $\begin{array}{c}70.00-13,000.00 \\
6,096 / 4,080 / 1,625 / 4 \\
\end{array}$ & $\begin{array}{c}3,067.0-3,190.0 \\
3,190 / 3,129 / 3,129 / 2 \\
\end{array}$ & $\begin{array}{c}146.00-175.00 \\
20.5 / 160.5 / 160.5 / 2 \\
\end{array}$ & $\begin{array}{c}180.00-333.00 \\
88.1 / 231.3 / 181 / 3 \\
\end{array}$ \\
\hline $\begin{array}{c}\text { Power } \\
\text { Capital Cost } \\
\end{array}$ & [US\$/KW] & $\begin{array}{c}0.00-10,200.00 \\
3,051 / 1,950 / 640 / 27\end{array}$ & $\begin{array}{l}15,000-125,000 \\
45 \mathrm{~K} / 71 \mathrm{~K} / 73 \mathrm{~K} / 4 \\
\end{array}$ & $\begin{array}{c}3,500.0-4,200.0 \\
495 / 3,850 / 3,850 / 2\end{array}$ & $\begin{array}{c}481.00-8,000.0 \\
3,263 / 3,130 / 1,170 / 5 \\
\end{array}$ \\
\hline \multicolumn{2}{|c|}{ Application } & $\begin{array}{l}\text { Small/Medium Scale } \\
\text { Energy Management }\end{array}$ & $\begin{array}{c}\text { Very Small Scale } \\
\text { Energy Management }\end{array}$ & $\begin{array}{c}\text { Medium Scale } \\
\text { Energy Management }\end{array}$ & $\begin{array}{c}\text { Medium Scale } \\
\text { Energy Management }\end{array}$ \\
\hline \multicolumn{2}{|c|}{ Technical Maturity } & $\begin{array}{c}\text { Proven/ } \\
\text { Commercializing }\end{array}$ & $\begin{array}{c}\text { Proven/ } \\
\text { Developing }\end{array}$ & $\begin{array}{c}\text { Proven/ } \\
\text { Developing }\end{array}$ & $\begin{array}{c}\text { Proven/ } \\
\text { Commercializing }\end{array}$ \\
\hline \multicolumn{2}{|c|}{ Environmental Impact } & Low & Low & Medium/Low & Medium/Low \\
\hline
\end{tabular}




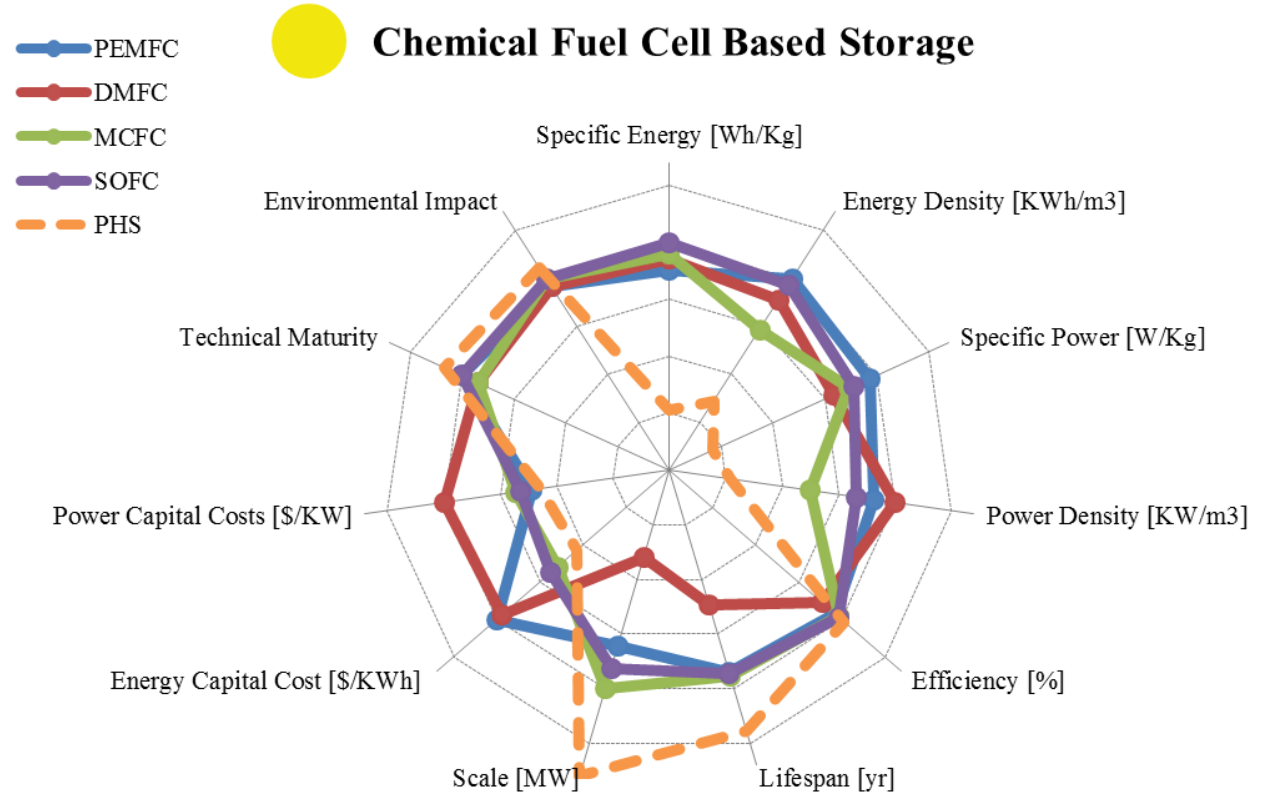

Figure 8. Comparing chemical storage and generation in fuel cells using normalized and logarithmically plotted average data from Table 7.

Proton exchange membrane fuel cells (PEMFC) are by far the most commonly discussed fuel cell for the portable market. These cells utilize direct conversion of hydrogen and oxygen for energy production. PEMFCs (and DMFCs) are among the only fuel cells that operate at low temperatures of around $70-85^{\circ} \mathrm{C}$. They are not capable of operating at sub-zero temperatures however. Devices are sometimes coupled with NiMH batteries to assist both in energy absorption (regenerative braking application) and to bring cell temperatures up for optimal performance [38]. PEMFCs, while more energy dense than Li-Ion chemistries, have slow startup times as well as expensive catalysts and membranes. Heat is usually needed to improve conduction and performance. Cells are extremely sensitive to impurities in oxygen and hydrogen and suffer from short lifespans. A shift in catalyst material has allowed the use of lower hydrogen content fuels. Smaller devices may even use air-breathing cells but are vulnerable to variations in environmental conditions-larger versions compensate for these issues at higher performance via forced air sub-systems. Most small cells are designed to utilize replaceable storage cartridges and so storage has been a significant research area [38]. Compressed gas, reversible metal hydrides, chemical hydrides and carbon based-hydrides have been considered. Most hydrides are safer and more energy dense than compressed gas variants, but require either higher temperatures or extremely low cryogenic temperatures for hydrogen release and retention. Recent advances in fabrication have leveraged the use of silicon chip and lithography manufacturing techniques to produce small flexible form factor devices. These microelectronic manufacturing techniques allow cheap production of storage hydrides, electrodes, electrolytes and membrane/catalyst layers for PEMFC. Despite improvements, to present day, commercial competitiveness of small fuel cells remains in question.

Direct methanol fuel cells (DMFC) are an alternative to existing PEMFCs for small portable applications. Like PEMFCs they can operate at low temperatures by converting liquid methanol (with potential water additive) directly to electricity. Manufacture of DMFCs can also leverage the microelectronics industry [38]. Unfortunately startup is sluggish even in these systems. Add to this methanol toxicity and low power density (similar to PEMFC) plus other PEMFC-like issues and 
development in this field remains far from commercial deployment [68]. Catalytic problems are the primary issue in these fuel cells. Traditional Plutonium, Palladium or Rhodium catalysts are not only expensive, but also exhibit slow kinetics and low efficiencies. Mesoporous nano-material with novel carbon supports have helped to improve performance. Explorations in aero/xerogels, carbon nano-fibres and nanotubes can reduce precious metal catalyst loading and improve efficiency [69]. The use of cheaper nickel and zirconium alloys as catalysts has been considered as an alternative too [68]. Higher membrane conductivities associated with acidic electrolytes makes them preferential. But these acidic electrolytes have significant corrosion effects meaning that a strong alkaline environment is needed. Unfortunately, strong alkalines causes carbonate formation and requires frequent electrolyte regeneration [68]. The use of acidic electrolytes separated by nanoporous silica has shown promise, but significant methanol crossover effects exist. Recent research has focused on reducing cell pressures and bringing temperatures down. Unfortunately, lower temperatures are associated with lower conduction in alkaline environments [70]. High temperature methanol can also cause membrane swelling [68]. All these issues suggest that DMFCs will require extensive research and development before they are ready for commercial market.

Molten carbonate fuel cells (MCFCs) move away from the small portable implementations to larger grid scale applications. These MCFC devices operate at temperatures in the range of about $600{ }^{\circ} \mathrm{C}$ and up. This means that hydrocarbon fuels can be converted and reformed to hydrogen rich gases via steam catalysis. Devices are resistant to impurities and unlikely to experience poisoning issues like those experienced by PEMFCs (and to lesser extent by DMFCs). Adding to this, the use of non-precious metal catalysts and they become suitable for large scale continuous use combined heat and power applications [38]. Electrodes for MCFCs must have high electrical conductivity, high mechanical resistance, good porosity and low solubility in molten electrolyte [71]. Recent advances have led to the use of oxidized nickel cathodes. Some replacement cobalt oxides have also been explored. Anode materials account for $25 \%$ of the cost of stacks and so new cheaper materials are needed. Addition of aluminium has been shown to improve mechanical resistance while addition of chromium stabilizes the porous anode structure against corrosion effects. Hot corrosion of anode, cathode and wet seals still has significant impact resulting in the loss of electrolyte and increased electrical resistance. Recent improvements have aimed at engineering micro and nano-scale structures as well as increasing dopants, coatings and additives to improve corrosion resistance and other performance characteristics. Hybridized molten carbonate and solid oxide approaches have also been attempted. Unfortunately, costs remain high and corrosion remains a problem [71].

Solid oxide fuel cells (SOFCs) have seen greater practical large scale applications than MCFCs. These fuel cells operate at approximately $1000{ }^{\circ} \mathrm{C}$ at high efficiencies using a non-corrosive metal oxide electrolyte [38]. These SOFCs are preferentially used in conjunction with combined heat and power (CHP) applications for greater efficiency. The high temperature of operation, however, increases costs, thermal stress and startup/shutdown times. As a result recent years have seen a shift to lower temperature SOFCs. Unfortunately, this is accompanied by a reduction in conductivity. Attempts to improve conductivity have involved the use of new electrolytes, thinner membrane layers, new fabrication techniques and nano-material dopings [72]. As with PEMFC, the use of hydrogen as a base fuel is a significant logistic challenge and so the use of SOFC has focused on reforming of hydrocarbon fuels (e.g., natural gas). Internal reforming is difficult however, so recent technologies have attempted direct 
hydrocarbon fuel cell development. The use of nickel based ceramic compounds for anode materials has shown potential for direct oxidation of hydrocarbons [72].

\subsection{Flow Batteries}

Flow batteries carry strong similarities to fuel cell devices. Electrolytes containing dissolved active materials flow through the fuel cell to generate electricity. There are two battery types, namely: Redox flow or metal/halide batteries. Redox flow batteries have two electrolyte storage tanks (containing catholyte and anolyte), a pumping system, and series/parallel connected bipolar cell stacks. Electrolyte colour changes are used to measure state of charge. Typically, these flow batteries are known for their long cycle life (under deep discharge) and their potential for large scale grid level storage. The energy and power independence and modularity of these redox batteries makes them particularly suited for a wide range of applications [73]. For these large scale storage options electrolyte costs are the primary cost predictors [74]. Metal/halide flow batteries are amongst the earliest implementations of flow batteries and utilize the deposition of metals as a means of storing energy. Table 8 and Figure 9 compares three particular variations of flow batteries.

Table 8. Comparing (chemical) flow batteries based on thirteen different metrics. Data obtained or calculated from multiple sources [7].

\begin{tabular}{|c|c|c|c|c|}
\hline \multirow{2}{*}{\multicolumn{2}{|c|}{ Metric }} & \multirow{3}{*}{$\begin{array}{c}\text { Vanadium Redox } \\
\text { range } \sigma / \boldsymbol{\mu} \widetilde{\boldsymbol{x}} / \boldsymbol{n} \\
10.00-50.00 \\
11.15 / 24.43 / 24.00 / 14 \\
\end{array}$} & \multirow{3}{*}{$\begin{array}{c}\text { Zinc Bromine } \\
\text { range } \boldsymbol{\sigma} / \boldsymbol{\mu} \widetilde{\boldsymbol{x}} / \boldsymbol{n} \\
11.10-90.00 \\
23.3 / 55.9 / 60.0 / 19\end{array}$} & \multirow{3}{*}{$\begin{array}{c}\text { Polysulphide Bromine } \\
\text { range } \boldsymbol{\sigma} / \boldsymbol{\mu} \widetilde{\boldsymbol{x}} / \boldsymbol{n} \\
10.00-50.00 \\
12.46 / 22.67 / 20.00 / 9 \\
\end{array}$} \\
\hline & & & & \\
\hline Specific Energy & {$[\mathrm{Wh} / \mathrm{Kg}]$} & & & \\
\hline Energy Density & {$\left[\mathrm{KWh} / \mathrm{m}^{3}\right]$} & $\begin{array}{c}10.00-33.00 \\
8.17 / 21.70 / 20.00 / 10\end{array}$ & $\begin{array}{c}5.17-70.00 \\
19.9 / 32.6 / 30.0 / 16\end{array}$ & $\begin{array}{c}10.80-60.00 \\
15.78 / 25.60 / 20.00 / 8\end{array}$ \\
\hline Specific Power & {$[\mathrm{W} / \mathrm{Kg}]$} & $\begin{array}{c}31.30-166.00 \\
54.89 / 110.46 / 125.00 / 5\end{array}$ & $\begin{array}{c}5.50-110.00 \\
37.2 / 56.9 / 60.0 / 9\end{array}$ & Unknown \\
\hline Power Density & {$\left[\mathrm{KW} / \mathrm{m}^{3}\right]$} & $\begin{array}{c}2.50-33.42 \\
21.86 / 17.96 / 17.96 / 2\end{array}$ & $\begin{array}{c}2.58-8.50 \\
2.60 / 5.87 / 6.00 / 5\end{array}$ & $\begin{array}{c}1.35-4.16 \\
1.99 / 2.76 / 2.76 / 2\end{array}$ \\
\hline Efficiency & {$[\%]$} & $\begin{array}{c}60.00-88.00 \\
7.28 / 76.96 / 78.30 / 19\end{array}$ & $\begin{array}{c}60.00-85.00 \\
7.21 / 73.3 / 73.0 / 17\end{array}$ & $\begin{array}{c}57.00-83.00 \\
8.65 / 71.45 / 75.00 / 11\end{array}$ \\
\hline Lifespan & {$[\mathrm{yr}]$} & $\begin{array}{c}2.00-20.00 \\
5.93 / 10.50 / 10.00 / 8\end{array}$ & $\begin{array}{c}5.00-20.00 \\
4.78 / 9.38 / 9.00 / 8\end{array}$ & $\begin{array}{c}10.00-15.00 \\
2.86 / 13.33 / 15.00 / 3\end{array}$ \\
\hline Cycle Life & [cycles] & $\begin{array}{c}800-16,000 \\
5,250 / 7,759 / 7,500 / 16\end{array}$ & $\begin{array}{c}800-5,000 \\
1,225 / 2,368 / 2,000 / 11\end{array}$ & $\begin{array}{c}800-4,000 \\
1,203 / 2,360 / 2,000 / 5\end{array}$ \\
\hline Scale & [MW] & $\begin{array}{c}0.00-20.00 \\
5.59 / 3.58 / 0.73 / 18\end{array}$ & $\begin{array}{c}0.001-20.00 \\
5.91 / 3.55 / 1.00 / 19\end{array}$ & $\begin{array}{c}0.001-100.00 \\
25.54 / 14.87 / 12.00 / 15\end{array}$ \\
\hline $\begin{array}{c}\text { Energy Capital } \\
\text { Cost }\end{array}$ & [US\$/KWh] & $\begin{array}{c}100.00-2,000.00 \\
542.4 / 488.1 / 200.0 / 15\end{array}$ & $\begin{array}{c}110.00-2,000.0 \\
525.6 / 447 / 225 / 13\end{array}$ & $\begin{array}{c}110.00-2,000.00 \\
559.05 / 494.08 / 187.50 / 16\end{array}$ \\
\hline $\begin{array}{c}\text { Power Capital } \\
\text { Cost }\end{array}$ & {$[\mathrm{US} \$ / \mathrm{KW}]$} & $\begin{array}{c}175.00-9,444.00 \\
2,355 / 2,461 / 1,545 / 15\end{array}$ & $\begin{array}{c}175.00-4,500.0 \\
1,365 / 1,788 / 1,300 / 12\end{array}$ & $\begin{array}{c}330.00-4,500.00 \\
1,208 / 1,643 / 1,098 / 12\end{array}$ \\
\hline Applica & & $\begin{array}{l}\text { Medium/Large Scale } \\
\text { Energy Management }\end{array}$ & $\begin{array}{c}\text { Large Scale } \\
\text { Energy Management }\end{array}$ & $\begin{array}{c}\text { Large Scale } \\
\text { Energy Management }\end{array}$ \\
\hline Technical N & urity & Proven/Commercializing & Proven/Developing & Proven/Developing \\
\hline Environment & Impact & Medium/Low & Medium & Medium \\
\hline
\end{tabular}




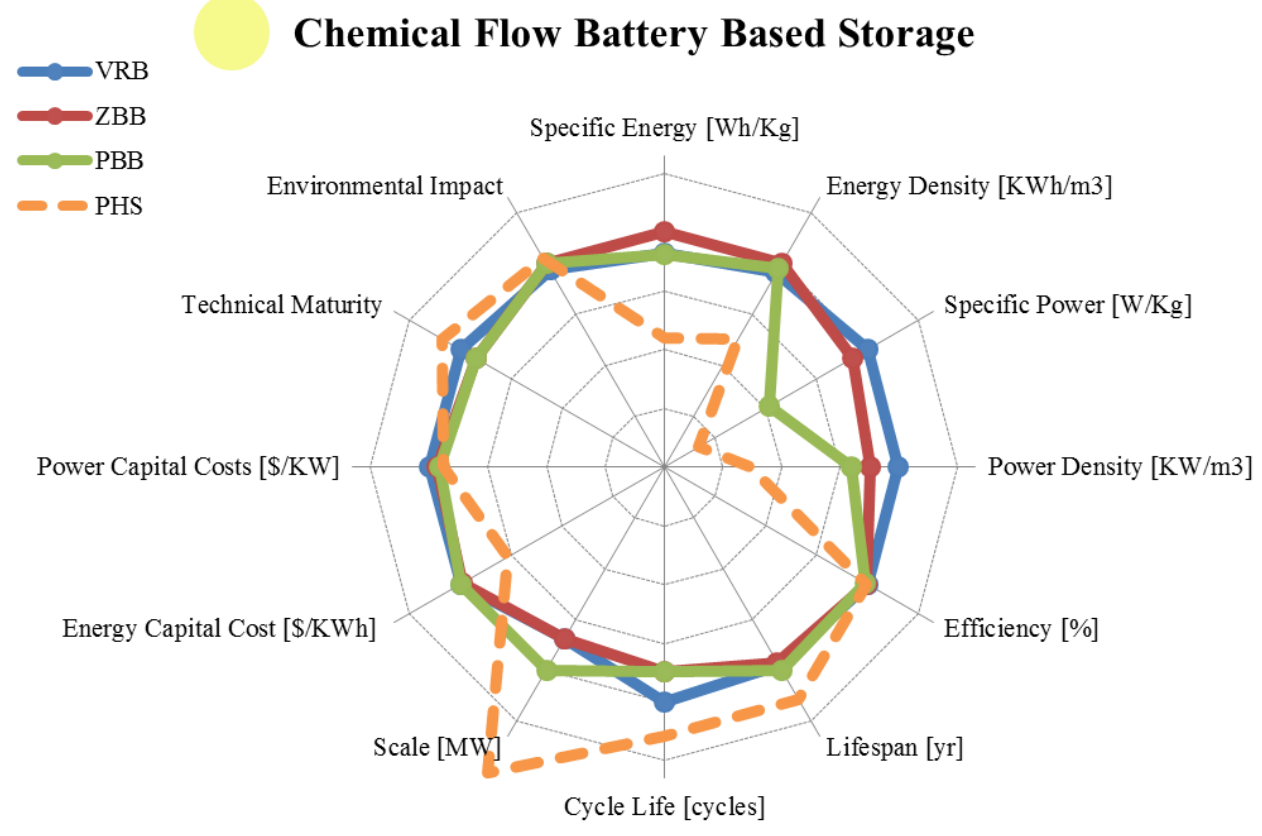

Figure 9. Comparing chemical storage and generation in flow batteries using normalized and logarithmically plotted data from Table 8.

A wide range of redox couples have been investigated. Among these are zinc bromine, polysulfide bromide, cerium zinc and all vanadium couples. Out of these, the most intensively explored chemistry has been the vanadium redox battery (VRB). These batteries utilize two ionic species of vanadium dissolved in the same electrolyte. They are well known for deep depth of discharge, long life, modularity, non-toxic materials and simplicity [73]. While these VRBs remain more expensive than lead acid batteries, they are quickly becoming cost competitive.

VRB energy densities also remain low, however, for large scale implementation they offer amongst the best storage options available to present day. The elimination of short circuit risks and active material shedding make them competitive with many larger scale chemical battery chemistries. Furthermore, electrolyte tanks can be embedded underground to reduce visual effects as well as effects of extreme climactic conditions. The separate tanks can be made out of a wide array of polymeric materials and eliminate the potential for sudden energy release as a result of electrolyte mixing [74]. The poor solubility of vanadium, however, has prompted the use of new electrolytes and the incorporation of stabilizing additives for supersaturated electrolytes. The discovery of graphene oxide, metallic catalyst and chemical treatment of electrodes has led to improvements in performance [75]. Membrane materials still need extensive development to deal with lower purity vanadium and withstand highly oxidizing environments. As with fuel cells, Nafion has been one such studied membrane. Polymer blending and inorganic blocking surface layers have been used to reduce permeation and cross-over of vanadium ions [75]. Experiments have also looked at cell stack designs that utilize zero spacing between membrane, electrodes and current collectors. Some have even looked at replacing one electrode with an air electrode to produce vanadium-oxygen redox batteries. It's worth noting that VRB systems, so far, are amongst the best choice when it comes to flow batteries.

Chemistries, such as polysulphide bromine (PBB) have been considered as an alternative because of the greater abundance of electrolyte materials and potential for lower cost. However, significant 
problems have been noted. These include problems of cross-contamination, electrolyte imbalances, membrane sulfur deposition and high safety risk resulting from corrosive bromine evolution. As a result, in practice, costs remain high [74]. Another bromine variant, the Zinc bromine (ZBB) based metal/halide battery has been amongst the earliest implementations of flow batteries. These ZBBs use the deposition of zinc and so energy capacity was traditionally limited and maintenance was high. The potential for high specific energy has been the main reason for continued research of ZBB systems. Like other flow batteries, they have high efficiency and deep discharge and utilize abundant and inexpensive materials. Unfortunately, despite research and improvements, the dendritic deposition of zinc lowers efficiencies and life. The high kinetics at high discharge rates result in eventual battery failure. Carbon electrodes have been used to improve performance to limited effect. The highly corrosive nature of bromine, as with PBB, also poses a safety risk and thus, combined with the other issues, commercialization of ZBB systems has been very limited [74].

\subsection{Final Remarks}

Most chemical energy storage systems have a number of common features, for instance: the electrodes, the electrolyte and the separators or membranes. Improvements have largely focused on materials. A shift has occurred towards more reactive electrodes. These more reactive variants have shown the promise of increasing energy and power densities - the use of lithium (e.g., Li-Ion batteries) and oxygen/air based chemistries (i.e., metal-air batteries) reflects this trend. Thin film, foam, fibre and polymer modifications (and material substitutions/additions) of existing electrodes have aimed at improved durability while benefiting performance. Virtually every chemical battery type has seen the utilization of nano-materials or sintering for further improvements. Hybridizations of different battery chemistries have attempted to combine the positive aspects of multiple chemistries while mitigating the negatives. Improvements in electrolyte have consisted of additives or substitutions designed to improve stability and ion exchange (conductivity). Electrolyte gelling, circulation and gas venting techniques provide a method for leak resistant, higher performance and safer batteries. Separator and membrane design improvements have focused on allowing ion exchange to occur without loss of active cell material. The durability of separators and membranes has been a significant issue in some battery chemistries and so these materials have also seen additions and polymerizations similar to those undergone by electrodes. The integration of microelectronics has been considered and modifications of charging regimes have been shown to greatly extend lifespan of many chemical storage solutions.

In the landscape of energy storage (i.e., mechanical, chemical, electromagnetic and thermal), chemical storage techniques remain the most well researched and well developed field. Most traditional chemical battery systems have excellent performance in one respect or another and have shown great suitability for specific small scale applications. However, they suffer from severe deficiencies for large scale storage. ZnAg batteries have excellent energy and power densities but suffer from short life and very high costs and so are only suitable for very small scale applications. ZnMn systems also have relatively short life but do better in terms of cost - these costs become high when attempting to deliver high power and so scale has been limited. For medium scale applications, Pb-Acid batteries have found a niche. These $\mathrm{Pb}$-Acid batteries are cheap and have high power delivery, however, they also have significant environmental implications and energy performance falls well below that of $\mathrm{ZnAg}$ and $\mathrm{ZnMn}$ 
chemistries. At a similar scale to Pb-Acid, Li-Ion chemistries have shown not only excellent energy and power performance, but also excellent cycle life. Li-Ion batteries, unfortunately, are expensive, have safety and charging issues and the shortage of lithium resources limits their applicability for any meaningful large scale system. Despite this, Li-Ion has displaced $\mathrm{NiMH}$ and $\mathrm{NiCd}$ systems. The abundance of materials, low cost, and very long life spans of NiFe systems have shown them as a potential larger scale storage solution. Unfortunately, these systems suffer from poor performance and high self-discharge rates. NiZn provides high performance at low cost but suffers from very short cycle life. Most traditional battery systems also suffer from permanently reduced life and capacities under very high discharge rates, improper storage environments, from overcharging or from complete discharge. Pre-mature failure is not uncommon with these batteries.

These clear deficiencies have led to the development of molten salt, metal-air, fuel cell and flow batteries. Both $\mathrm{NaS}$ and $\mathrm{NaNiCl}$ molten salt systems show very good energy and power performance and high suitability for grid level large scale applications. These systems are approaching cost competitiveness with traditional chemical battery systems and while more expensive than both PHS and CAES, might be worthwhile investments given their significantly higher performance. Metal-air systems such as Zn-Air and Fe-Air have been restricted to small scale application. However, their extremely high performance and low costs may make them even better alternatives than molten-salt batteries if cycle life and higher discharge rates can be achieved. Some metal-air batteries have shown performances approaching those of burnt hydrocarbon fuels. Fuel cells offer an alternative to burning, have high energy and power performance and have seen some commercial applications to large scale grid level storage/generation. Unfortunately, they remain on shaky ground, like metal-air chemistries, as a result of their poor lifespans, high costs and potential high operating temperatures. Adding to this is the problem of hydrogen storage, fuel reforming and the potential for moderate emissions. Combined with PHS, CAES, fuel cells and molten salt batteries, flow batteries may also be immediate contenders for grid level storage - these batteries, not only have long low maintenance life but relatively high power and energy densities too. There is need, however, to reduce costs even further to be competitive with other solutions. Though it's too early to tell, it's likely that in the short term future, there will be a shift towards CAES storage as well as molten salt and flow battery storage. In the slightly longer term, fuel cells may also become more prevalent. Traditional chemical batteries are unlikely to be strong contenders for large scale storage at this point, though with additional research metal-air chemistries may hold future promise.

\section{Electromagnetic Storage}

Traditionally, electromagnetic storage was limited to capacitors and inductors. Recent material advances have allowed the development of supercapacitors and superconductors and has extended the use of capacitive and inductive technologies to larger scale applications. Table 9 and Figure 10 provides a summary of performance characteristics of both kinds of device. 
Table 9. Comparing electromagnetic storage based on fourteen different metrics. Data obtained or calculated from multiple sources [7].

\begin{tabular}{|c|c|c|c|}
\hline \multirow{2}{*}{\multicolumn{2}{|c|}{ Metric }} & Superconducting & Supercapacitor \\
\hline & & range $\sigma / \mu / \widetilde{x} / n$ & range $\sigma / \mu / \tilde{x} / n$ \\
\hline Specific Energy & {$[\mathrm{Wh} / \mathrm{Kg}]$} & $\begin{array}{c}0.27-75.00 \\
21.43 / 11.79 / 5.00 / 11\end{array}$ & $\begin{array}{c}0.07-85.60 \\
20.62 / 16.36 / 10.00 / 25\end{array}$ \\
\hline Energy Density & {$\left[\mathrm{KWh} / \mathrm{m}^{3}\right]$} & $\begin{array}{c}0.20-13.80 \\
4.45 / 4.99 / 3.85 / 12\end{array}$ & $\begin{array}{c}1.00-35.00 \\
11.81 / 13.77 / 10.00 / 9\end{array}$ \\
\hline Specific Power & {$[\mathrm{W} / \mathrm{Kg}]$} & $\begin{array}{c}500.00-15,000.00 \\
6,570.77 / 5,600.00 / 2,000.00 / 5\end{array}$ & $\begin{array}{c}5.44-100,000.00 \\
20,154.00 / 8,930.44 / 3,500.00 / 24\end{array}$ \\
\hline Power Density & {$\left[\mathrm{KW} / \mathrm{m}^{3}\right]$} & $\begin{array}{c}300.00-4,000.00 \\
1,719.85 / 1,457.50 / 765.00 / 4\end{array}$ & $\begin{array}{c}15.00-4,500.00 \\
2,000.74 / 921.00 / 30.00 / 5\end{array}$ \\
\hline Efficiency & {$[\%]$} & $\begin{array}{c}80.00-99.00 \\
5.72 / 92.45 / 95.00 / 11 \\
\end{array}$ & $\begin{array}{c}65.00-99.00 \\
9.63 / 91.33 / 95.00 / 12 \\
\end{array}$ \\
\hline Lifespan & {$[\mathrm{yr}]$} & $\begin{array}{c}20.00-30.00 \\
5.77 / 25.00 / 25.00 / 4 \\
\end{array}$ & $\begin{array}{c}5.00-20.00 \\
5.35 / 11.43 / 10.00 / 7 \\
\end{array}$ \\
\hline Cycle Life & [cycles] & $\begin{array}{c}10,000-100,000 \\
40,865.63 / 68,000.00 / 90,000.00 / 5\end{array}$ & $\begin{array}{c}10,000-1,000,000 \\
364,649 / 302,308 / 100,000 / 13\end{array}$ \\
\hline Self-Discharge Rate & {$[\% /$ day $]$} & $\begin{array}{c}1.00-15.00 \\
6.25 / 7.50 / 7.00 / 3\end{array}$ & $\begin{array}{c}0.46-40.00 \\
16.43 / 18.64 / 20.00 / 7\end{array}$ \\
\hline Scale & {$[\mathrm{MW}]$} & $\begin{array}{c}0.01-200.00 \\
48.55 / 23.56 / 8.00 / 22\end{array}$ & $\begin{array}{c}0.00-5.00 \\
1.29 / 0.52 / 0.02 / 23\end{array}$ \\
\hline Energy Capital Cost & [US\$/KWh] & $\begin{array}{c}500.00-1,080,000.00 \\
336,835 / 125,488 / 2,755 / 10 \\
\end{array}$ & $\begin{array}{c}100.00-94,000.00 \\
28,160 / 19,866 / 9,750 / 16 \\
\end{array}$ \\
\hline Power Capital Cost & [US\$/KW] & $\begin{array}{c}196.00-10,000.00 \\
2,412.70 / 981.56 / 325.00 / 16\end{array}$ & $\begin{array}{c}100.00-800.00 \\
226.46 / 321.00 / 300.00 / 10\end{array}$ \\
\hline \multicolumn{2}{|c|}{ Application } & $\begin{array}{c}\text { Medium/Large Scale } \\
\text { Power Quality }\end{array}$ & $\begin{array}{c}\text { Small/Medium Scale } \\
\text { Power Quality }\end{array}$ \\
\hline \multicolumn{2}{|c|}{ Technical Maturity } & Proven/Commercializing & Proven/Commercializing \\
\hline \multicolumn{2}{|c|}{ Environmental Impact } & Low & Very Low \\
\hline
\end{tabular}

\subsection{Superconducting Magnetic Energy Storage}

Superconductors have seen a range of storage/generation applications. They have been used in flywheel storage systems for low friction bearings, in synchronous generators for improved generating performance and in fusion power systems to assist with plasma containment [76]. However, superconducting magnetic energy storage (SMES) refers to their use for energy storage in the magnetic field of an inductor. As with FES, the main application of SMES has been for short term power quality and stability - this came about from the observation that $90 \%$ of grid failures are sags and outages lasting less than one second but are responsible for costly damages.

Superconductors have very long lifespans, cycle life, high efficiency, fast response and very high discharge rates [77]. This power capacity is not matched by energy performance and energy capital costs remain very high. There are four main components associated with SMES: The superconductor, the refrigeration, the containment vessel and the power converter [78]. Micro-SMES systems have seen significant commercial deployment. These systems combine all four components into a single black box 
solution [78]. Utilities, however, remain cautious about adopting these new technologies. The long lead times suggests that sustained government and partner roles are needed to progress SMES and emphasis on parallel development of related cryogenic and converter technologies is needed [76]. Hybrid solutions have aimed at combining SMES with traditional Pb-Acid UPS systems whereby the superconductor provides short term high discharge and reduces initial high discharge loading on Pb-Acid batteries [78]. Alternative systems have attempted to use liquid hydrogen SMES combined with fuel cells. Their use has begun to extend to onsite wind energy applications too. SMES is widely regarded as environmentally friendly, however, some concern remains over safety and health risks associated with high magnetic fields.

\section{Electromagnetic Energy Storage}

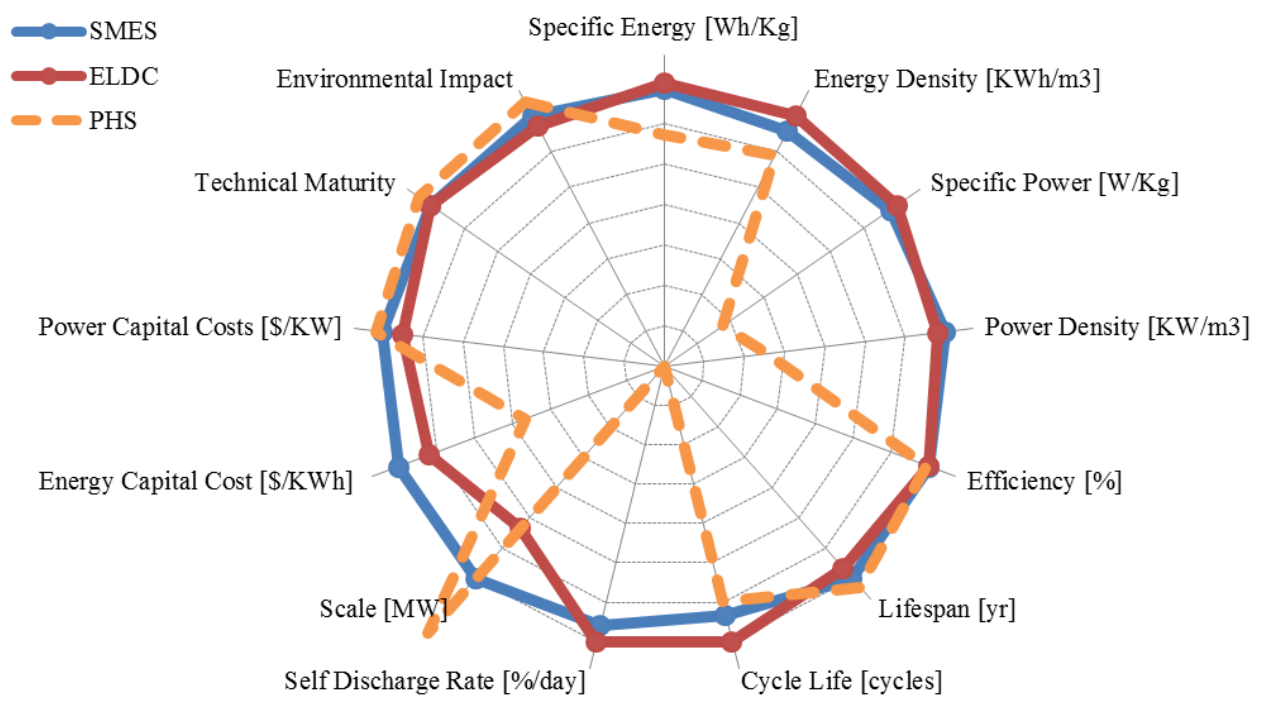

Figure 10. Comparing electromagnetic storage using normalized and logarithmically plotted average data from Table 9.

Super conductors in SMES are typically either solenoidal or toroidal in topology. Solenoidal topologies have more stray magnetic fields. Toroidal approaches reduce these stray fields at the expense of mechanical complexity. These topologies are cooled to a critical temperature depending on the type of superconductor - metal alloys such as nobium titanium or nobium tin that operate at low temperatures at or below $-249{ }^{\circ} \mathrm{C}$ were used in the past. The rising cost of helium and the discovery of ceramic oxide-based superconductors have ushered in the use of high temperature variants operating at up to $-138^{\circ} \mathrm{C}$ and allowing the use of cheaper liquid nitrogen [76]. The cost of superconductors is likely to always remain higher than copper conductors so cost benefits must be quite significant to justify their use. Recent efforts have focused on topology changes to minimize superconductor use [79]. The development of superconducting materials has, however, followed the exponential trend of transistors and other similar technologies so cost and performance improvements are likely to occur [76]. It should be noted that cryogenics and power control and conversion are the major barriers to adoption not the superconductors themselves [78].

The development of superconductors has occurred in parallel with cryogenic cooling systems. These systems originally used external vapour cooled liquefier systems, but recent advances have seen the use 
thermo-acoustic (pulse tube) cyro-cooling [76,78]. Cooling costs can be lowered with the use of high temperature superconductors. Cooling times can be long and size and cost reduction of cooling systems is still a significant challenge, as is reliability. With the low temperatures, the need for safe containment is also a consideration and can add to system size.

Power conditioning and control remain a challenge in SMES. The development of variable speed ac motor drives has lent considerable progress to power conversion [78]. Typical SMES systems utilize either thyristor, voltage source or current source converters. Power conditioning systems (PCS) handle power transfer between SMES and ac grid and operate similar to flywheel circuits. The incorporation of SMES into existing flexible ac transmission system infrastructure can reduce costs associated with grid interconnection significantly.

\subsection{Supercapacitor Energy Storage}

Electrostatic capacitors utilize electrodes separated by a dielectric to store energy in an electric field. Unfortunately, they have low capacitance making them unsuitable for higher power applications. Some improvements result from the use of electrolytes as with electrolytic capacitors. But these still have limitations. Supercapacitors are electrochemical double layer capacitors (EDLC) that use a combination of porous separators and electrolyte in place of a dielectric. Electrolytes can be organic or aqueous. These capacitors operate at low voltages but have high capacitance, extremely long cycle life, wide operating temperatures and high discharge rates. As a result, these ELDC products have seen a number of commercial applications. These applications have looked at memory backup, battery support for high discharge environments and to provide grid stability. Unfortunately, supercapacitors suffer from high parasitic losses, relatively low energy performance and high cost. In addition the low voltages require series stacking. The stacking results in unequal voltage distributions among ELDC cells and reduces lifespan and performance [80].

Supercapacitors can be categorized as symmetric or asymmetric (hybrid) based on whether or not both electrodes are the same material or different materials. Many of the developments from chemical battery systems are applicable to supercapacitors. The utilization of carbon foams, fibres, aerogels, xerogels, nanotubes and the use of conductive polymers and metal oxides has shown significant improvements in performance. Some electrodes may be composites. Tangled networks with open central channels have shown exceptional specific capacitance. The volumetric reactions resulting from polymer film electrodes have shown further performance benefits over the surface reactions of traditional carbon electrodes. Metal oxides remain easy to manufacture while nanotubes are largely within the arena of research [80]. Higher voltages can be achieved by organic electrolytes but these are accompanied by higher resistivity. Aqueous electrolytes provide better conductivity at the cost of reduced breakdown voltage. Separators allow ion exchange via the electrolyte but prevent electrode short circuiting. Problems of unequal voltage distribution can be handled by passive or active voltage equalization circuits. Passive approaches for smaller scale applications have concentrated on resistive bypass or zener diode voltage regulation. A shift has been seen towards zener approaches because of the lower losses. These passive approaches are substituted for active equalizing current systems for high duty cycle low parasitic loss applications. These active approaches may take the form of buck-boost style controls [80]. 


\subsection{Final Remarks}

Both SMES and ELDC solutions hold excellent promise in power quality applications. These solutions, unfortunately, both have low energy performance. The development of nanotubes and nano-particles are providing continued improvements in ELDC energy performance. Both SMES and ELDC fall within the same scope as FES systems, with FES systems continuing to offer better energy performance than both ELDC and SMES.

\section{Thermal Storage}

Thermal storage systems are not new and have been used extensively in home temperature stabilization as well as industrial scale thermal power plants and district heating applications. The mechanical, chemical and electromagnetic storage solutions discussed in previous section of this review have focused on electricity storage. The use of thermal energy storage, however, must be coupled to some form of thermoelectric generator or heat engine for electricity generation. As such numerical comparison of real thermal storage with other forms of storage is difficult. This difficulty is compounded by the fact that most reported numbers relate to theoretical heat storage capacities of materials rather than real values for functioning systems that produce electricity - both Table 10 and Figure 11 attempt to report some figures, however these should not be taken as accurate. It is recommended that future research look at specific products and systems in more detail and report figures in terms of volumetric and gravimetric energy and power densities from practical installations coupled with power generation facilities. Despite the lack of sufficient data, when comparing systems, the most important considerations appear to be the thermal capacity of the materials, phase change temperature, thermal conductivity/stability, abundance and cost [81].

The use of thermal storage and heat recovery has allowed power plant efficiencies to increase up-to $60 \%$ for natural gas plants. The advent of solar thermal systems has further encouraged development in the field. Most solar thermal plants are located in the USA and Spain and typically take one or more of the following forms: parabolic troughs, dish, fresnel or heliostat systems [81]. There are suggestions that with appropriate policies to accelerate development, as much as $50 \%$ of the heating needs of Europe could be supplied by thermal systems by 2030 [82].

There are three types of storage that have emerged: traditional sensible heat storage (STES), latent heat storage (LTES) and reversible chemical reaction heat storage (CTES). Systems normally contain three main components: the thermal material, the heat exchanger and the containment system. These are sometimes coupled with steam accumulators used to stabilize steam availability in thermal plants.

\subsection{Sensible Heat Storage}

By far the most ubiquitous form of storage is sensible heat thermal energy storage (STES). These are materials such as concretes, salts, metals and fire bricks that absorb heat in the order of $0.85-1.15 \mathrm{KJ} / \mathrm{Kg}$ at specific temperatures-i.e., the specific heat capacity. The heat is absorbed and released when the ambient surrounds fall below the material temperature. There are two main forms of STES systems: Passive and active. In passive systems the storage medium is fixed and heat is transferred through a passive heat transfer mechanism or fluid. In active systems the heat storage medium itself is circulated. 
In active direct systems separate tanks hold the hot and the cold storage material. These systems require no additional heat exchanger and heat transfer occurs quickly, however they have higher costs. With active indirect systems both the hot and cold storage occur in the same tank - these indirect systems are somewhat cheaper and require carefully controlled charge and discharge to maintain stratification. Some systems employ silica or quartz to assist in maintaining the thermocline for improved stratification [81]. In general, heat storage materials for STES are either solid or liquid. Concrete and fire bricks are heavily used solid forms, and, among liquids, molten salts are cheap, abundant and have good heat capacities and are often used for active storage from solar concentrator systems. However salts can potentially freeze. For lower temperatures ( 0 to $100{ }^{\circ} \mathrm{C}$ ), applications involving water are amongst the best with capacities well above many other materials. Alternatives have included the use of oils and organic liquids [81]. STES systems can be hybridized with latent heat storage for improved performance.

Table 10. Comparing thermal storage based on fourteen different metrics. Unlike previous comparisons values associated with thermal storage don't take into account electricity generation. Data obtained or calculated from multiple sources [7].

\begin{tabular}{|c|c|c|c|c|}
\hline \multirow{2}{*}{\multicolumn{2}{|c|}{ Metric }} & Sensible Heat & Latent Heat & Reaction Heat \\
\hline & & range $\sigma / \mu / \tilde{x} / n$ & $\operatorname{range} \sigma / \mu / \tilde{x} / n$ & range $\sigma / \mu \widetilde{x} / n$ \\
\hline Specific Energy & {$[\mathrm{Wh} / \mathrm{Kg}]$} & $\begin{array}{c}10.00-120.00 \\
46.55 / 65.00 / 65.00 / 4\end{array}$ & $\begin{array}{c}150.00-250.00 \\
52.5 / 209.3 / 227.8 / 3\end{array}$ & $\begin{array}{c}250.00 \\
\mathrm{x} / 250.00 / 250.00 / 1\end{array}$ \\
\hline Energy Density & {$\left[\mathrm{KWh} / \mathrm{m}^{3}\right]$} & $\begin{array}{c}25.00-120.00 \\
34.57 / 77.00 / 80.00 / 5\end{array}$ & $\begin{array}{c}100.00-370.00 \\
122.9 / 197.5 / 160.0 / 4\end{array}$ & $\begin{array}{c}300.00 \\
\times / 300.00 / 300.00 / 1\end{array}$ \\
\hline Specific Power & {$[\mathrm{W} / \mathrm{Kg}]$} & Unknown & $\begin{array}{c}10.00-30.00 \\
14.14 / 20.00 / 20.00 / 2\end{array}$ & Unknown \\
\hline Power Density & {$\left[\mathrm{KW} / \mathrm{m}^{3}\right]$} & Unknown & Unknown & Unknown \\
\hline Efficiency & {$[\%]$} & $\begin{array}{c}7.00-90.00 \\
34.34 / 51.75 / 55.00 / 4\end{array}$ & $\begin{array}{c}75.00-90.00 \\
10.61 / 82.50 / 82.50 / 2\end{array}$ & $\begin{array}{c}75.00-100.00 \\
17.68 / 87.50 / 87.50 / 2\end{array}$ \\
\hline Lifespan & {$[\mathrm{yr}]$} & $\begin{array}{c}10.00-20.00 \\
7.07 / 15.00 / 15.00 / 2\end{array}$ & $\begin{array}{c}20.00-40.00 \\
14.14 / 30.00 / 30.00 / 2\end{array}$ & Unknown \\
\hline Cycle Life & [cycles] & Unknown & Unknown & Unknown \\
\hline Self-Discharge Rate & [\%/day $]$ & $\begin{array}{c}0.50 \\
\mathrm{x} / 0.50 / 0.50 / 1 \\
\end{array}$ & $\begin{array}{c}0.50-1.00 \\
0.35 / 0.75 / 0.75 / 2 \\
\end{array}$ & Unknown \\
\hline Scale & {$[\mathrm{MW}]$} & $\begin{array}{c}0.001-10.00 \\
7.07 / 5.00 / 5.00 / 2\end{array}$ & $\begin{array}{c}0.001-300.00 \\
149.82 / 75.28 / 0.55 / 4\end{array}$ & $\begin{array}{c}0.01-1.00 \\
0.70 / 0.51 / 0.51 / 2\end{array}$ \\
\hline Energy Capital Cost & {$[\mathrm{US} \$ / \mathrm{KWh}]$} & $\begin{array}{c}0.04-50.00 \\
17.42 / 11.06 / 2.26 / 8 \\
\end{array}$ & $\begin{array}{c}3.00-88.73 \\
836.52 / 40.73 / 30.00 / 5 \\
\end{array}$ & $\begin{array}{c}10.90-137.00 \\
88.84 / 73.74 / 73.74 / 2\end{array}$ \\
\hline Power Capital Cost & [US\$/KW] & $\begin{array}{c}2,500.00-7,900.00 \\
2,844 / 4,683 / 3,650 / 3\end{array}$ & $\begin{array}{c}200.00-300.00 \\
70.7 / 250.0 / 250.0 / 2\end{array}$ & Unknown \\
\hline \multicolumn{2}{|c|}{ Application } & $\begin{array}{l}\text { Medium Scale } \\
\text { Bridging Power }\end{array}$ & $\begin{array}{l}\text { Medium/Large Scale } \\
\text { Energy Management }\end{array}$ & $\begin{array}{l}\text { Small/Medium Scale } \\
\text { Energy Management }\end{array}$ \\
\hline \multicolumn{2}{|c|}{ Technical Maturity } & Mature/Commercialized & Proven/Commercializing & Proven/Developing \\
\hline \multicolumn{2}{|c|}{ Environmental Impact } & Very Low & Low/Uncertain & Low/Uncertain \\
\hline
\end{tabular}




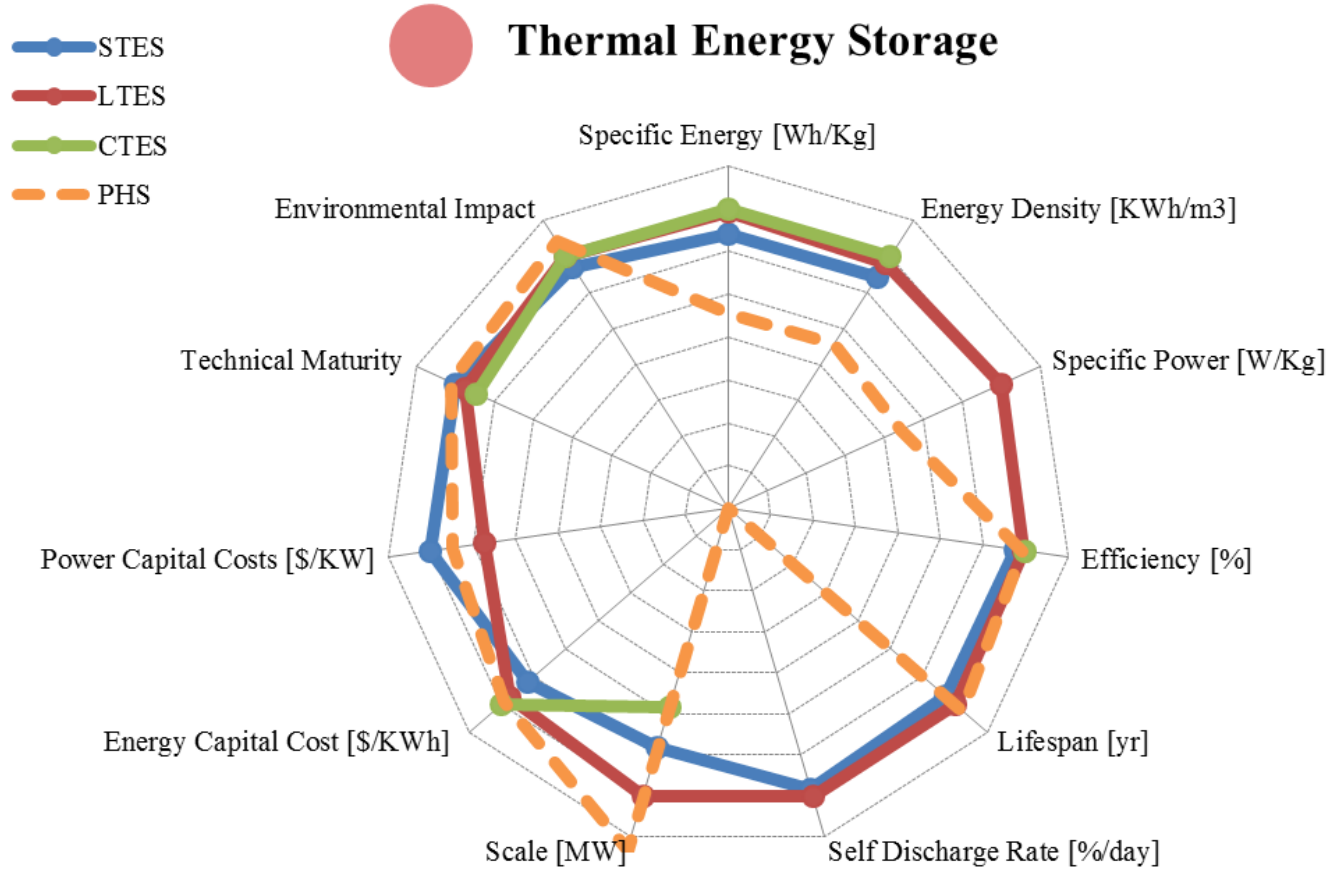

Figure 11. Comparing thermal storage using normalized and logarithmically plotted average data from Table 10.

\subsection{Latent Heat Storage}

Unlike STES, latent heat thermal energy storage (LTES) utilizes the heat absorbed during phase transition (the heat of fusion) for energy storage. There are three types of materials commonly used: organic, inorganic and eutectic.

Organic paraffin appears to be quite popular, but non-paraffin materials are far more numerous and have various desirable properties. Organic materials are also more expensive but are non-corrosive, chemically stable and easy to incorporate into building materials. Inorganic materials include salt hydrates and metallic compounds. Salt hydrates, while widely explored, suffer from super-cooling issues and low thermal conductivities both of which can detract from system performance. Metallic and graphite additives have been used to improve conductivity in these hydrates [81]. Inorganic materials are generally cheaper than organic materials, but suffer from chemical decomposition after repeated cycling. Eutectic materials are composite mixture of materials that have phase transition at lower temperatures than their constituent materials thus allowing fine tuning of storage temperatures while taking advantage of the benefits of both organic and inorganic materials [83]. LTES systems tend to have higher energy storage potential than STES systems. Heat of fusion in these systems can range from $100-340 \mathrm{KJ} / \mathrm{Kg}$ at operational temperatures - that's about two orders of magnitude bigger than STES materials [84]. LTES is often synonymous to phase transition materials (PCM). The materials can undergo four main transitions: solid-liquid, solid-solid, solid-gas and liquid-gas. In terms of flexibility and easy containment (from reduced volume change) solid-solid transition materials are very attractive and do significantly better than sensible storage in concrete. Solid-gas transitions, however, offer the best storage capacity but also undergo the largest volume change during transition resulting in containment issues. As a compromise between storage capacity and ease of containment, most PCMs utilize solid-liquid phase transitions. 
Commercially available PCMs usually take the form of macro-capsule solid pellets usually containing an appropriately selected wax. To improve construction material compatibility and transportability micro-capsules are used in wet slurry, paste or powder form. Sometimes, PCM material is absorbed, post-manufacture, into plasterboards and other construction materials directly. Both macro- and micro-capsules have applications ranging not only from construction but to clothing, bedding, food curing, drying and plant growth, thermal and solar thermal energy generation, solar cooking and even to electronics as well [83]. Unfortunately, unlike, STES, most PCMs can't be used for heat transfer as well, so a separate heat exchange mechanism is needed (e.g., heat pipes). Utilization of PCMs for improved heating and cooling has shown huge improvements in fuel efficiency and promises reductions in generation capacity via large scale distributed peak shaving [83].

\subsection{Reversible Chemical Reaction Heat Storage}

Reversible chemical reaction heat thermal energy storage (CTES) provides a third and more energy dense and efficient storage medium than both STES and LTES. There are three main types of CTES: heat pump systems, heat pipe systems, heat of reaction systems [81,83]. Chemical heat pumps (CHP) utilize adsorption (exothermic) and desorption (endothermic) of a vapour/liquid onto a solid substance. Typically materials for CHP come in working pairs (e.g., metal hydrides-hydrogen, hydrates-water, ammoniates-ammonia, etc.). Chemical heat pipes (CHPi) are very similar to both CHPs and traditional heat pipes but utilize the dissociation of aqueous solutions of acid or bases (e.g., sodium hydroxide) as a mechanism for thermal storage. Chemical reaction heat (CRH) systems operate by breaking down compounds into their constituent parts via an endothermic process and by releasing that heat and remixing the individual components [85]. These reversible chemical systems are very much in their infancy and significant research is currently underway. However, they are extremely interesting in that they can also enable the low loss transport of waste heat. As such reversible chemical approaches offer the potential for long term storage not available from other thermal forms. Metal hydride systems are usually made from nickel, iron, magnesium or cobalt alloys combined with hydrogen. Pelletized versions have been considered [81]. Salt hydrates are similar but lock water in crystalline form. These hydrates unfortunately are slow and have unpredictable kinetics. The combination of careful temperature and pressure control and the use of additives can improve the kinetics. Some approaches utilize solid-gas pairs. One such example is the use of ice with carbon dioxide [86]. This approach may have relevance when combined with carbon sequestration.

\subsection{Final Remarks}

Sensible storage is by far the cheapest form of thermal storage and is ideally suited for short term applications. For longer term or more energy dense storage latent heat storage offers greater potential. Reversible chemical reaction heat is the most promising and least developed form of thermal storage. In real systems it is likely that a combination of all three storage forms will be used. Thermal storage has strong potential for existing power plants and solar thermal installations and can significantly increase energy efficiencies and reduce losses for existing systems. This is especially true in the context of energy storage in molten salt batteries, fuel cells and even compressed air systems. 


\section{Conclusions}

The landscape of energy storage is extensive. This review has discussed 27 types of storage technologies. Some storage technologies are strongly coupled to particular generation technologies. For instance, PHS systems show strong similarities with hydro-electric plants and are often used in conjunction with nuclear facilities. CAES systems are much like peaking gas turbine plants. Fuel cells, as they increase in scale operate much like traditional thermal generation plants, albeit converting fuel directly to electricity. This is true of some flow battery configurations too. Thermal storage systems are integral parts of thermal (and solar thermal) plants and are often used in the context of steam generation and waste heat recovery for subsequent power plant cycles.

Storage technologies have been compared numerically and qualitatively on the basis of fourteen parameters, namely: specific energy, energy density, specific power, power density, efficiency, lifespan, cycle life, self-discharge rate, scale, energy capital cost, power capital cost, application, technical maturity and environmental impact. Within each of these categories and parameters, numbers have been obtained from a variety of sources where possible. The number of sources sampled (n) and their rough distribution provides not only the numerical range over which devices operate but also the standard deviation $(\sigma)$, average $(\mu)$ and medial $(\tilde{x})$ parameter values for the particular device. Device parameters that go less reported in literature are typically correlated with low sample numbers. It is recommended that a unified database of storage and generation technologies be constructed. To this end, the numerical data associated with each device (along with the source) has been included with this paper in [7].

Figure 12 provides a summary of energy and power performances across the entire spectrum of storage solutions considered in this review. As noted earlier, PHS accounts for $99 \%$ of worldwide deployed energy storage, yet it is the least energy and power dense solution of all the storage options. The low loss storage capacities of PHS installations have lent them to easy adoption for large scale energy management applications.
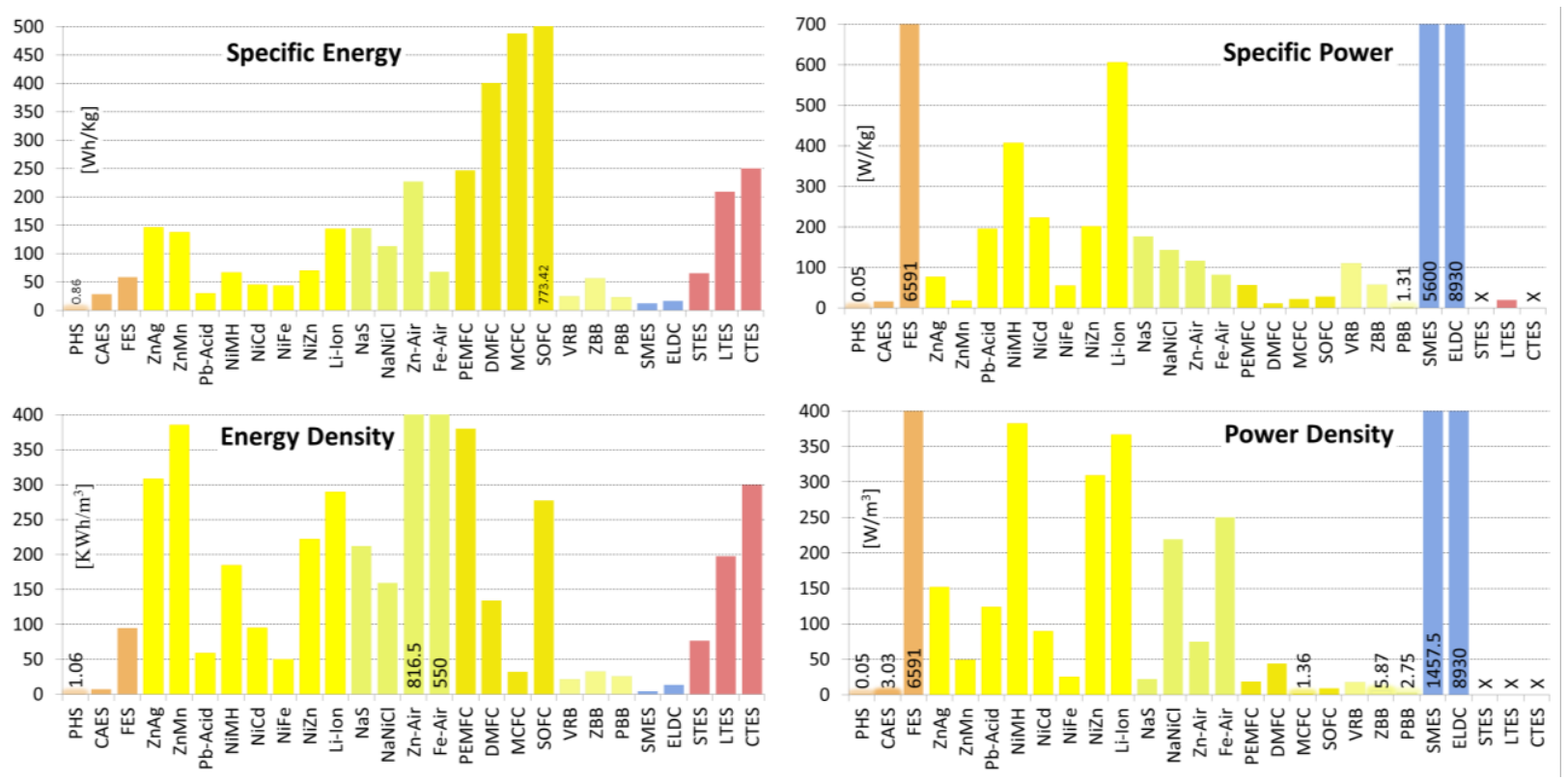

Figure 12. Energy and power performance of storage devices with respect to each other. Note that data points with "X" marks are unknown. 
With increasing concerns over energy security and sustainability, as well as a decreased availability of storage sites and low profitability of PHS, recent years have seen the development of a host of new options. Of these, among the most viable contender for large-scale grid level storage is CAES. CAES offers greater deployment locations and higher energy and power capacities. Furthermore it has low capital costs, low losses and long life (see Figures 13 and 14).
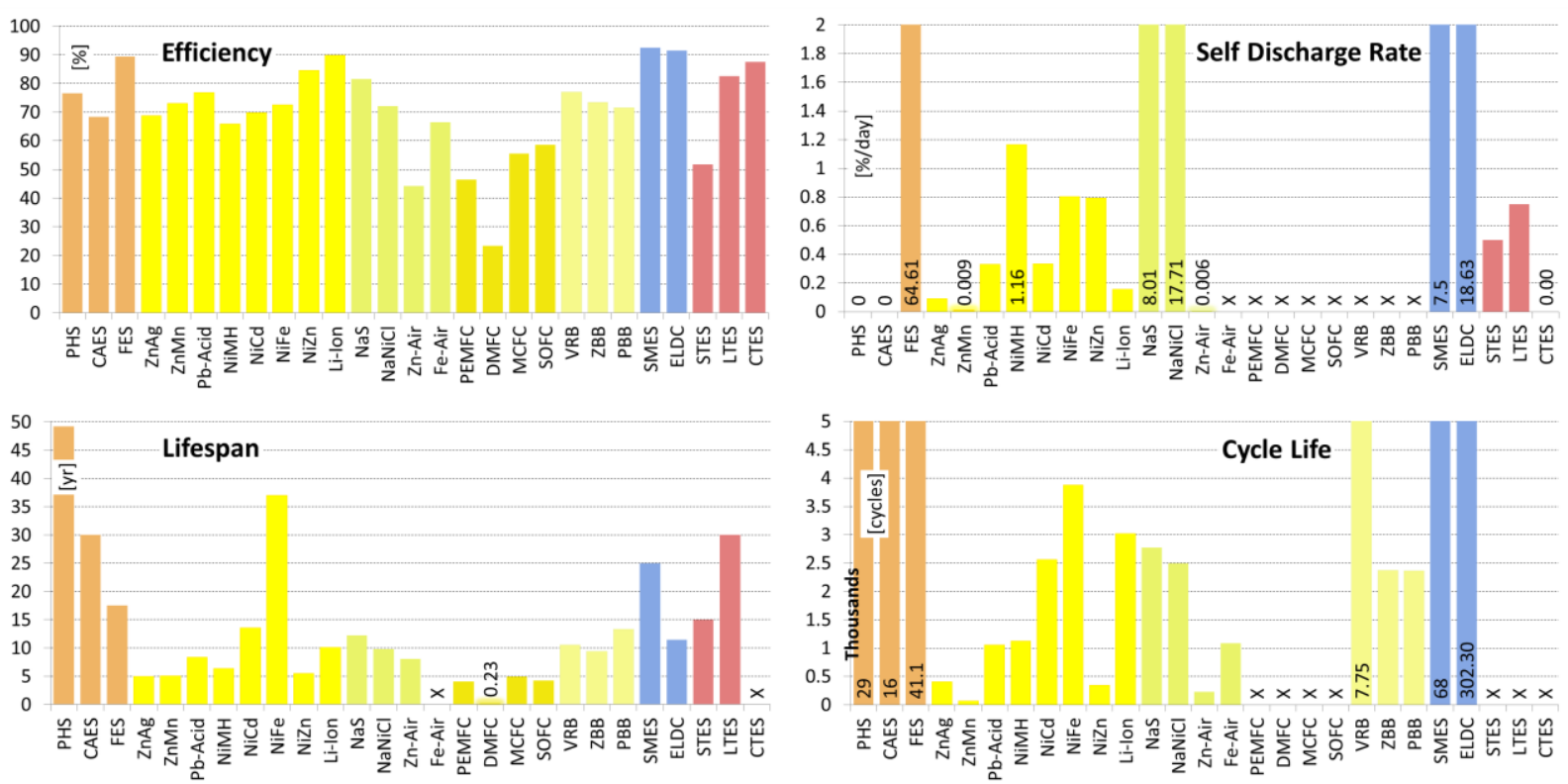

Figure 13. Comparing storage effectiveness across storage devices. Data points marked with "X" are unknown.
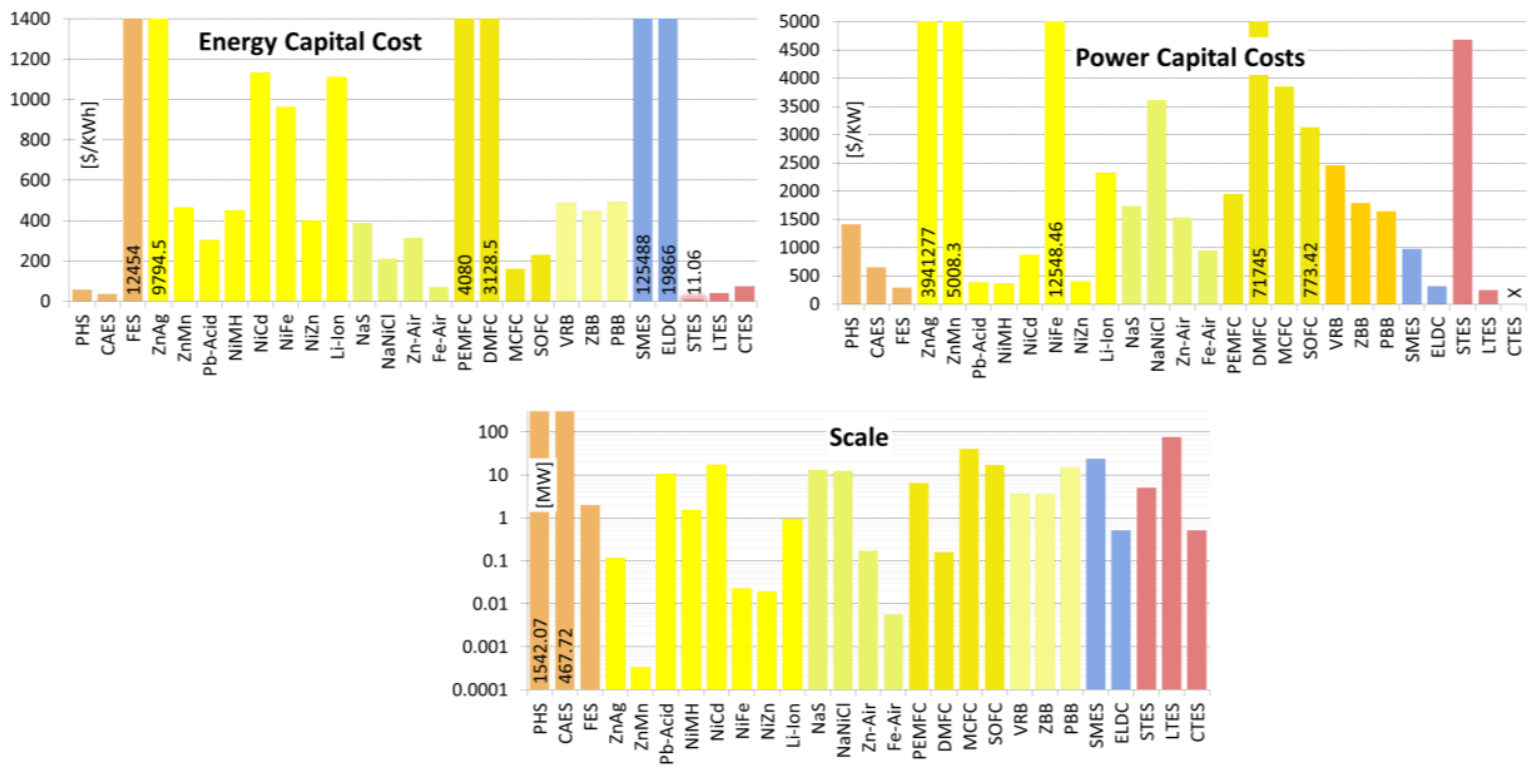

Figure 14. Comparing cost and scale of technologies with respect to each other. Data points marked with "X" are unknown.

Following CAES, flow batteries appear to be another large scale solution for storage. These batteries suggest the possibility of improved energy and power performance over CAES systems, meaning that systems can, potentially, be decoupled from specialized geologically suitable sites. Unfortunately, 
flow batteries have high costs and potentially higher environmental and safety impacts (see Figure 15). Deployments of flow battery systems have been relatively small scale and significant effort is needed for commercialization.
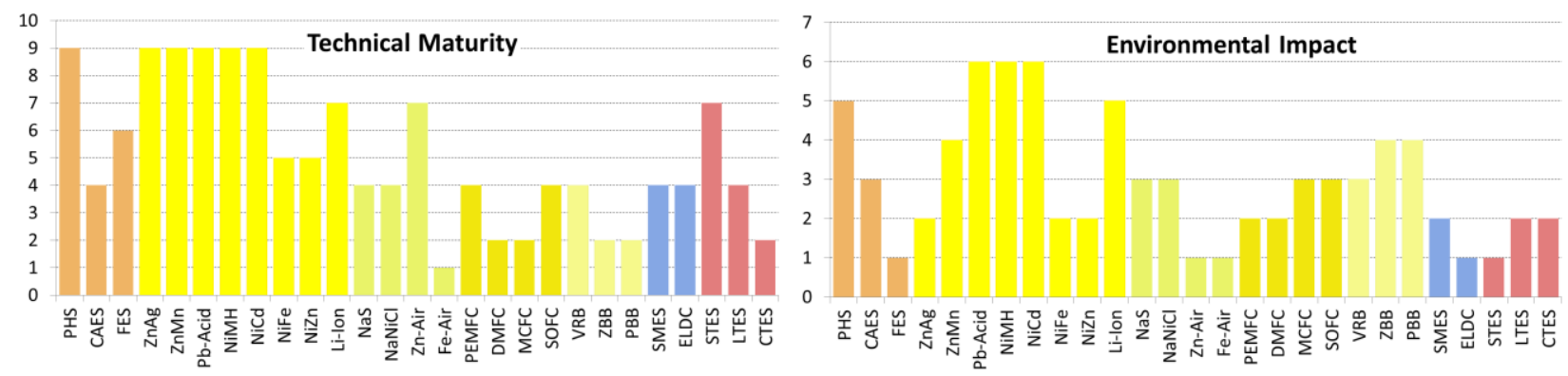

\begin{tabular}{|l|l|l|}
\hline \multicolumn{2}{|l|}{ Technical Maturity Scale Conversion } \\
\hline Very Mature & Fully Commercialized & 9 \\
\hline Very Mature & Commercialized & 8 \\
\hline Mature & Commercialized & 7 \\
\hline Mature & Commercializing & 6 \\
\hline Mature & Limited Development & 5 \\
\hline Proven & Commercializing & 4 \\
\hline Proven & Limited Development & 3 \\
\hline Proven & Developing & 2 \\
\hline Research & Developing & 1 \\
\hline
\end{tabular}

\begin{tabular}{|l|l|}
\hline Environmental Impact Scale Conversion \\
\hline Very Very High & 8 \\
\hline Very High & 7 \\
\hline High & 6 \\
\hline High / Medium & 5 \\
\hline Medium & 4 \\
\hline Medium / Low & 3 \\
\hline Low & 2 \\
\hline Very Low & 1 \\
\hline
\end{tabular}

Figure 15. Comparing qualitative metrics: technical maturity and environmental impact. Data points marked with " $X$ " are unknown.

After flow batteries, fuel cells also offer high potential for large scale storage/generation. Of-course, as with other generation technologies, separate fuel storage and transportation considerations are required. The energy performance of fuel cells is far in excess of PHS, CAES and flow batteries. This means a significant reduction of land usage. Among, fuel cells the most promising appear to be high temperature SOFC style devices - these have seen some commercial deployment. Unfortunately, fuel cells suffer from major lifespan related issues - until these have been addressed, it is unlikely that the technology will be a major player in large or small scale storage. Metal-air batteries bear a lot of similarity to fuel cells and show higher power performance but remain in the very early stages of development as well.

Barring fuel cells and metal-air batteries, at the smaller scale, a number of contenders present themselves. Molten salt, $\mathrm{NaS}$ and $\mathrm{NaNiCl}$ systems have seen applications in electric vehicles and exhibit reasonable power and energy performance as well as long life. These devices are easy to recycle, rugged and well suited for heavy and light duty charge/discharge regimes. They also hold promise for larger scale applications and may be better suited (as a result of longer life) than fuel cells to meet this challenge. They are, however, high temperature devices that require thermal management and experience related parasitic losses.

As an alternative requiring simpler thermal management, Li-Ion batteries, while not suited for large grid level applications (due to resource availability) have begun to see significant promise for electric vehicles as well. While it remains to be seen which chemistry will be successful at the smaller scales, far more extensive research efforts have been made in the direction of lithium chemistries as opposed to molten salt chemistries. This suggests that Li-Ion is likely to displace $\mathrm{NaS}$ and $\mathrm{NaNiCl}$ for electric vehicle applications. Li-Ion batteries have been remarkably successful and have displaced or are 
displacing NiCd and NiMH batteries in this arena. Likewise, NiZn chemistries traditional used in electric scooters have also undergone similar displacement - primarily due to their short cycle life.

An additional final contender for smaller scale storage, especially in the context of renewable installations appears to be the NiFe battery. This chemistry is rugged, long lived, environmentally benign, easily maintained, easily charged and discharged and has the potential to be of lower cost than Li-Ion chemistries. Unfortunately, after its displacement by $\mathrm{Pb}$-Acid, this chemistry has seen little commercial deployment and so development must catch up significantly before the batteries become competitive.

Remaining battery chemistries such as $\mathrm{ZnMn}, \mathrm{ZnAg}$ are still largely restricted much smaller scale applications. Moving away from electricity storage, we see that thermal energy storage systems are becoming a major research area especially in the context of efficiency improvements in existing generation infrastructure. They have been applied to solar thermal generation and have significant implications for distributed and district heating applications. The development of all three: STES, LTES and CTES, is likely to occur in parallel with other storage (e.g., molten salt, fuel cell, compressed air) and generation systems. Furthermore, in the context of management and regulation of grid power quality, FES, ELDC and SMES systems have found a niche. Power delivery of these three technologies is unparalleled to any other system and it's likely that all three will continue to improve in parallel to other "energy management" focused storage solutions. However, parasitic losses have prevented their direct application to energy management thus far.

From large scale to small scale, in Figure 16, we can see a more complete picture of the particular region of application for each of the storage methods discussed in this review. The next few years promise to be exciting ones and will, see technologies mature and find their own respective niches.

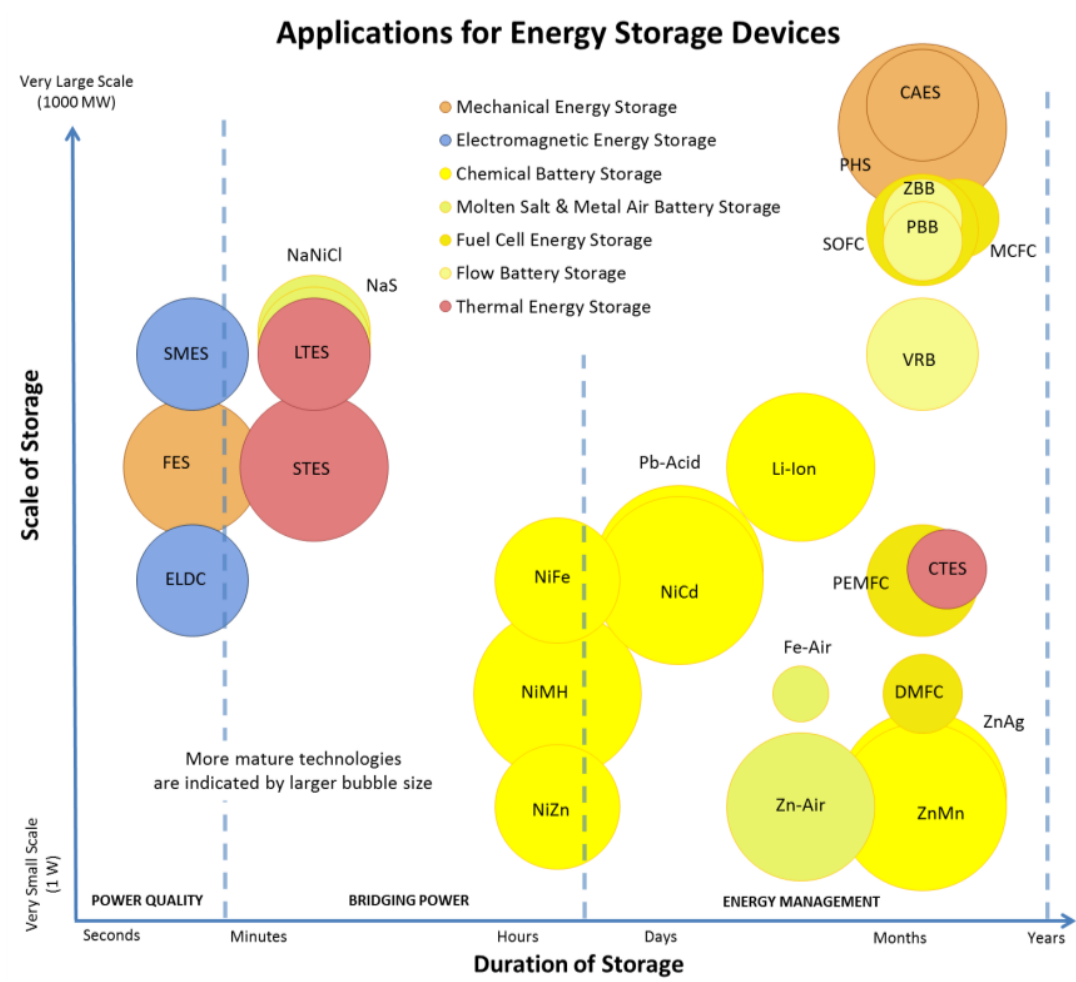

Figure 16. A rough breakdown of the specific applications for which storage technologies are suited. Detailed sub-divisions of these applications are provided in Tables 1 and 2. For fuel cells hydrogen storage is assumed. Larger bubbles indicate more mature technologies. 


\section{Acknowledgments}

This work was supported by the Principal Career Development Scholarship (PCDS). Special acknowledgements to Daniel Friedrich, Anna Dunbar, Dimitri Mignard and Joseph William Burchell (the all powerful and all knowing) for providing editorial comments.

\section{Author Contributions}

Siraj Sabihuddin compiled numerical tables and graphs and completed writing of this paper. The writing was reviewed by Aristides Kiprakis and Markus Mueller.

\section{Conflicts of Interest}

The authors declare no conflict of interest.

\section{References}

1. Shafiee, S.; Topal, E. When will fossil fuel reserves be diminished. Energy Policy 2009, 37, 181-189.

2. Worldwide Trends in Energy Use and Efficiency: Key Insights from Iea Indicator Analysis; International Energy Agency (IEA): Paris, France, 2008.

3. International Energy Outlook 2013; U.S. Energy Information Administration: Washington, DC, USA, 2013.

4. Chen, H.; Cong, T.N.; Yang, W.; Tan, C. Progress in electrical energy storage system: A critical review. Prog. Nat. Sci. 2009, 19, 291-312.

5. Key World Energy Statistics; International Energy Agency (IEA): Paris, France, 2013.

6. Renewables 2013: Global Status Report; Renewable Energy Policy Network for the 21st Century: Paris, France, 2013.

7. Sabihuddin, S. A numerical and graphical review of energy storage and energy production technologies (dataset). Available online: http://figshare.com/articles/A_Numerical_and_Graphical_ Review_of_Energy_Storage_and_Energy_Production_Technologies/1096289 (accessed on 18 November 2014).

8. Bueno, C.; Carta, J.A. Wind powered pumped hydro storage systems, a means of increasing the penetration of renewable energy in the canary islands. Renew. Sustain. Energy Rev. 2006, 10, 312-340.

9. Deane, J.P.; Gallacho, B.P.O.; McKeogh, E.J. Techno-economic review of existing and new pumped hydro energy storage plant. Renew. Sustain. Energy Rev. 2010, 14, 1293-1302.

10. Pickard, W.F. The history, present state, and future prospects of underground pumped hydro formassive energy storage. Proc. IEEE 2012, 100, 473-483.

11. Yang, C.-J.; Jackson, R.B. Opportunities and barriers to pumped-hydro energy storage in the united states. Renew. Sustain. Energy Rev. 2011, 15, 839-844.

12. Steffen, B. Prospects for pumped-hydro storage in germany. Energy Policy 2012, 45, 420-429.

13. Droste-Franke, B. Assessing environmetal impacts of storage technologies and competing options for balanacing demand and supply in 2050. In Proceedings of the International Renewable Energy Storage Conference (IRES), Berlin, Germany, 12 November 2012. 
14. Harano, M.; Tani, K.; Nomoto, S. Practical application of high-performance Francis-Turbine runner fitted with splitter blades at Ontake and Shinkurobegawa No. 3 power stations of the Kansai Electric Power Co., Inc. Hitachi Rev. 2006, 55, 109-113.

15. Bierbower, W. Unconventional "hydraulic hydro storage" system offers energy storage for the grid on a grande scale. Available online: http://blogs.worldwatch.org/revolt/unconventional-"hydraulichydro-storage"-system-offers-energy-storage-for-the-grid-on-a-grand-scale/ (accessed on 18 November 2014).

16. Study of Compressed Air Energy Storage with Grid and Photovoltaic Energy Generation; Arizona Research Institute for Solar Energy (AZRISE): Tucson, AZ, USA, 2010.

17. Saadat, M.; Li, P.Y. Modeling and control of a novel compressed air energy storage system for offshore wind turbine. In Proceedings of the American Control Conference, Montreal, QC, Canada, 27-29 June 2012.

18. Lund, H.; Salgi, G. The role of compressed air energy storage (caes) in future sustainable energy systems. Energy Convers. Manag. 2009, 50, 1172-1179.

19. Compressed Air Energy Storage Power Plants; Bine Informationsdienst: Bonn, Germany, 2007.

20. Qin, C.; Loth, E. Liquid piston compression efficiency with droplet heat transfer. Appl. Energy 2014, 114, 539-550.

21. Ven, J.D.V.D.; Li, P.Y. Liquid piston gas compression. Appl. Energy 2009, 86, 2183-2191.

22. Advanced adiabatic compressed air energy storage (AA-CAES). Available online: $\mathrm{http}$ ://energystorage.org/advanced-adiabatic-compressed-air-energy-storage-aa-caes (accessed on 18 November 2014).

23. Pena-Alzola, R.; Sebastian, R.; Quesada, J.; Colmenar, A. Review of flywheel based energy storage systems. In Proceedings of the International Conference on Power ENgineering, Energy and Electrical Drives, Malaga, Spain, 11-13 May 2011.

24. Bolund, B.; Bernhoff, H.; Leijon, M. Flywheel energy and power storage systems. Renew. Sustain. Energy Rev. 2007, 11, 235-258.

25. Hebner, R.; Pratap, S.; Lewis, M.; Hearn, C. Low-Cost Flywheel Energy Storage for Mitigating the Variability of Renewable Power Generation; The University of Texas at Austin: Austin, TX, USA, 2012.

26. Liu, H.; Jiang, J. Flywheel energy storage-An upswing technology for energy sustainability. Energy Build. 2007, 39, 599-604.

27. Ragheb, M. Kinetic Energy Flywheel Energy Storage; University of Illinois at Urbana-Champaign: Champaign, IL, USA, 2013.

28. Koyanagi, J. Durability of filament-wound composite flywheel rotors. Mech. Time-Depend. Mater. 2012, 16, 71-83.

29. Strasik, M.; Johnson, P.E.; Day, A.C.; Mittleider, J.; Higgins, M.D.; Edwards, J.; Schindler, J.R.; McCrary, K.E.; McIver, C.R.; Carlson, D.; et al. Design, fabrication, and test of a 5-kwh/100-kw flywheel energy storage utilizing a high-temperature superconducting bearing. IEEE Trans. Appl. Supercond. 2007, 12, 2133-2138.

30. Sabihuddin, S.; Aristides Kiprakis, M.M. A diamagnetically stabilized magnetically levitated flywheel battery. In Proceedings of the Ecologic Vehicles Renewable Energies (EVER), Monte Carlo, The Principality of Monaco, 27-30 March 2013. 
31. Siebert, M.; Ebihara, B.; Jansen, R.; Fusaro, R.L.; Morales, W.; Kascak, A.; Kenny, A. A Passive Magnetic Bearing Flywheel; NASA: Savannah, GA, USA, 2002.

32. Praveen, R.P.; Ravichandran, M.H.; Achari, V.T.S.; Raj, V.P.J.; Madhu, G.; Bindu, G.R. Design and analysis of zero cogging brushless dc motor for spacecraft applications. In Proceedings of the International Conference on Electrical Engineering, Chaing Mai, Thailand, 19-21 May 2010.

33. Lam, N.M.; Huyen, N.T.T.; Manh, D.H.; Ky, V.H.; Tung, D.K.; Dan, N.H. Fabrication of Nd-Fe-B exchange-spring magnets. J. Phys.: Conf. Ser. 2009, 187, 1-5.

34. Rolim, L.G.B.; Ferreira, A.C.; Sotelo, G.G.; de Andrade, R., Jr. Flywheel generator with switched reluctance machine. In Proceedings of the International Conference on Electrical Machines (ICEM), Rio de Janeiro, Brazil, 6-9 October 2002.

35. Suvorkova, E.; Vajda, I.; Dementiev, Y. Electromagnetic design of hybrid synchronous motor. In Proceedings of the International Symposium PARNU: Topical Problems in the Field of Electrical and Power Engineering, Seoul, Korea, 8-11 October 2013.

36. Hansen, J.G.R.; O'Kain, D.U. An Assessment of Flywheel High Power Energy Storage Technology for Hybrid Vehicles; Oak Ridge National Laboratory: Oak Ridge, TN, USA, 2011.

37. Tarascon, J.-M.; Armand, M. Issues and challenges facing rechargeable lithium batteries. Nature 2001, 414, 359-367.

38. Linden, D.; Reddy, T.B. Handbook of Batteries; McGraw-Hill: New York, NY, USA, 2002.

39. Dueber, R. Strategic overview of silver-zinc rechargeaable batteries. Battery Power Prod. Technol. 2008, 12, 1-2.

40. Olivetti, E.; Gregory, J.; Kirchain, R. Life Cycle Impacts of Alkaline Batteries with A Focus on End-of-Life; Massachusetts Institute of Technology: Cambridge, MA, USA, 2011.

41. Huang, Y.-J.; Lin, Y.-L.; Li, W.-S. Manganese dioxide with high specific surface area for alkaline battery. Chem. Res. Chin. Univ. 2012, 28, 874-877.

42. Ghaemi, M.; Amrollahi, R.; Ataherian, F.; Kassaee, M.Z. New advances on bipolar rechargeable alkaline manganese dioxide-zinc batteries. J. Power Sources 2003, 117, 233-241.

43. Zhu, Z.; Tam, T.K.; Sun, F.; You, C.; Zhang, Y.-H.P. A high-energy-density sugar biobattery based on a synthetic enzymatic pathway. Nat. Commun. 2014, 4, doi:10.1038/ncomms4026.

44. Kincaid, B.; Poynter, B.; Braam, K.; Taniguchi, H. Printable Zinc-Manganese Dioxide Batteries; University of California Berkeley: Berkeley, CA, USA, 2010.

45. Evans, J.W.; Keist, J.; Ho, C.; Quan, B.; Wright, P.K. Printed Energy Storage; University of California Berkeley: Berkeley, CA, USA, 2014.

46. Banerjee, S. Low-Cost Grid-Scale Electrical Storage Using a Flow-Assisted Rechargeable ZincManganese Dioxide Battery; The City University of New York: New York, NY, USA, 2012.

47. Buchmann, I. Battery University. Available online: http://batteryuniversity.com/ (accessed on 18 November 2014).

48. Xu, J.; Thomas, H.R.; Francis, R.W.; Lum, K.R.; Wang, J.; Liang, B. A review of the processes and technologies for the recycling of lithium-ion secondary batteries. J. Power Sources 2008, 177, 512-527.

49. Cyrus Wadia, P.A. Venkat Srinivasan. Resource constraints onthe battery energy storage potential for grid and transporation applications. J. Power Sources 2011, 196, 1593-1598. 
50. Ritchie, A.; Howard, W. Recent developments and likely advances in lithium-ion batteries. J. Power Sources 2006, 162, 809-812.

51. Fetcenko, M.A.; Ovshinsky, S.R.; Reichman, B.; Young, K.; Fierro, C.; Koch, J.; Zallen, A.; Mays, W.; Ouchi, T. Recent advances in nimh battery technology. J. Power Sources 2007, 165, 544-551.

52. Bauerlein, P.; Antonius, C.; Loffler, J.; Kumpers, J. Progress in high-power nickel-metal hydride batteries. J. Power Sources 2008, 176, 547-554.

53. Tsai, P.-J.; Chan, S.L.I. Nickel-based batteries: Materials and chemistry. In Electricity Transmission, Distribution and Storage Systems; Woodhead Publishing Limited: New Delhi, India, 2013.

54. Bradwell, D.J. Liquid Metal Batteries: Ambipolar Electrolysis and Alkaline Earth Electroalloying Cells; Massachusetts Institute of Technology (MIT): Cambridge, MA, USA, 2011.

55. Hatta, T. Recent Applications of nas Battery System in the United States and in Japan; Sandia National Laboratories: Albuquerque, NM, USA, 2011.

56. Tanaka, K.; Kurashima, Y.; Tamakoshi, T. Recent Sodium Sulfur Battery Applications in Japan; In Proceedings of the IEEE/PES Transmission and Distribution Conference and Exposition, Atlanta, GA, USA, 28 October-2 November 2001; pp. 1169-1173.

57. Min, J.K.; Lee, C.-H. Numerical study on the thermal management system of a molten sodium-sulfur battery module. J. Power Sources 2012, 210, 101-109.

58. Benson, D.K.; Potter, T.F.; Tracy, C.E. Design of a Variable-Conductance Vacuum Insulation; National Renewable Energy Laboratory (NREL): Detroit, MI, USA, 1994.

59. Burch, S.D.; Parish, R.C.; Keyser, M.A. Thermal Management of Batteries Using a Variable-Conductance Insulation Enclosure; National Renewable Energy Laboratory (NREL): Orlando, FL, USA, 1995.

60. Ellis, B.L.; Nazar, L.F. Sodium and sodium-ion energy storage batteries. Curr. Opin. Solid State Mater. Sci. 2012, 16, 168-177.

61. Wen, Z.; Cao, J.; Gu, Z.; Xu, X.; Zhang, F.; Lin, Z. Research on sodium sulfur battery for energy storage. Solid State Ionics 2008, 179, 1697-1701.

62. Zyl, A.V. Review of the zebra battery system development. Solid State Ionics 1996, 86-88, 883-889.

63. Lee, J.-S.; Kim, S.T.; Cao, R.; Choi, N.-S.; Liu, M.; Lee, K.T.; Cho, J. Metal-air batteries with high energy density: Li-air versus zn-air. Adv. Energy Mater. 2011, 1, 34-50.

64. Koretz, B.; Harats, Y.; Goldstein, J. Operational aspects of the electric fuel zinc-air battery system for evs. In Proceedings of the 12th International Seminar on Primary and Secondary Battery Technology and Application, Deerfield Beach, FL, USA, 6-9 March 1995.

65. Cooper, J. Powering future vehicles with the refuelable zinc/air battery. Sci. Technol. Rev. 1995, October, 6-13.

66. Manohar, A.K.; Malkhandi, S.; Yang, B.; Yang, C.; Prakash, G.K.S.; Narayanan, S.R. A high-performance rechargeable iron electrode for large-scale battery-based energy storage. J. Electrochem. Soc. 2012, 159, A1209-A1214.

67. Cheng, X.; Shi, Z.; Glass, N.; Zhang, L.; Zhang, J.; Song, D.; Liu, Z.-S.; Wang, H.; Shen, J. A review of pem hydrogen fuel cell contamination: Impacts, mechanisms, and mitigation. J. Power Sources 2007, 165, 739-756.

68. Aricò, A.S.; Baglio, V.; Antonucci, V. Direct methanol fuel cells: History, status and perspectives. In Electrocatalysis of Direct Methanol Fuel Cells; Wiley-VCH Verlag: Darmstadt, Germany, 2009. 
69. Basri, S.; Kamarudin, S.K.; Daud, W.R.W.; Yaakub, Z. Nanocatalyst for direct methanol fuel cell (dmfc). Int. J. Hydrog. Energy 2010, 35, 7957-7970.

70. Ren, X.; Zelenay, P.; Thomas, S.; Davey, J.; Gottesfeld, S. Recent advances in direct methanol fuel cells at los alamos national laboratory. J. Power Sources 2000, 86, 111-116.

71. Cassir, M.; Ringuede, A.; Lair, V. Molten carbonates from fuel cells to new energy devices. In Molten Salt Chemistry; Elsevier: Burlington, MA, USA, 2013.

72. Zhao, Y.; Xia, C.; Jia, L.; Wang, Z.; Li, H.; Yu, J.; Li, Y. Recent progress on solid oxide fuel cell: Lowering temperature and utilizing non-hydrogen fuels. Int. J. Hydrog. Energy 2013, 38, 16498-16517.

73. Blanc, C.; Rufer, A. Understanding the vanadium redox flow batteries. In Paths to Sustainable Energy; InTech: Glasgow, UK, 2010.

74. Skyllas-Kazacos, M.; Menictas, C. Redox flow batteries for medium- to large-scale energy storage. In Electricity Transmission, Distribution and Storage Systems; Woodhead Publishing Limited: Cambridge, MA, USA, 2013.

75. Parasuramana, A.; Lima, T.M.; Menictasc, C.; Skyllas-Kazacos, M. Review of material research and development for vanadium redox flow battery applications. Electrochim. Acta 2013, 101, 27-40.

76. Superconductivity: Present and Future Applications. Available online: http://www.ccas-web.org/ pdf/ccas_brochure_web.pdf (accessed on 25 December 2014).

77. Ali, M.H.; Wu, B.; Dougal, R.A. An overview of smes applications in power and enegy systems. IEEE Trans. Sustain. Energy 2010, 1, 38-47.

78. Buckles, W.; Hassenzahl, W.V. Superconducting magnetic energy storage. Power Eng. Rev. 2000, May, 16-20.

79. Dai, T.; Tang, Y.; Shi, J.; Jiao, F.; Wang, L. Design of a $10 \mathrm{mj}$ hts superconducting magnetic energy storage magnet. IEEE Trans. Appl. Supercond. 2010, 20, 1356-1359.

80. Sharma, P.; Bhatti, T.S. A review on electrochemical double-layer capacitors. Energy Convers. Manag. 2010, 51, 2901-2912.

81. Muthukumar, P. Thermal energy storage: Methods and materials. Indian Institute of Technology Guwahati: Sevilla, Spain, 2011.

82. Kerskes, H.; Mette, B.; Bertsch, F.; Asenbeck, S.; Drück, H. Chemical energy storage using reversible solid/gas-reactions (cws) - results of the research project. Energy Procedia 2012, 30, 294-304.

83. Sharma, A.; Tyagi, V.V.; Chen, C.R.; Buddhi, D. Review on thermal energy storage with phase change materials and applications. Renew. Sustain. Energy Rev. 2009, 13, 318-345.

84. Farid, M.M.; Khudhair, A.M.; Razack, S.A.K.; Al-Hallaj, S. A review on phase change energy storage: Materials and applications. Energy Convers. Manag. 2004, 45, 1597-1615.

85. Thermal Energy Satorage: Technology Brief; International Renewable Energy Agency (IRENA): Abu Dhabi, UAE, 2013.

86. Fischer, M.; Bruzzano, S.; Egenolf-Jonkmanns, B.; Zeidler-Fandrich, B.; Wack, H.; Deerberg, G. Thermal storage by thermoreversible chemical reaction systems. Energy Procedia 2014, 48, 327-336.

(C) 2014 by the authors; licensee MDPI, Basel, Switzerland. This article is an open access article distributed under the terms and conditions of the Creative Commons Attribution license (http://creativecommons.org/licenses/by/4.0/). 